\title{
Pillar[5]arene-Modified Gold Nanorods as Nanocarriers for Multi-Modal Imaging-Guided Synergistic \\ Photodynamic-Photothermal Therapy
}

\author{
Nan Song, ${ }^{\dagger, \S}$ Zhijun Zhang, ${ }^{\dagger, \S}$ Peiying Liu, ${ }^{\dagger}$ Dihua Dai, ${ }^{\perp}$ Chao Chen, ${ }^{\#}$ Youmei Li, ${ }^{\dagger, \S}$ Lei Wang, ${ }^{\dagger} \S$ Ting \\ Han ${ }^{\dagger}$ Ying-Wei Yang, ${ }^{\perp, *}$ Dong Wang, ${ }^{\dagger}, *$ and Ben Zhong Tang ${ }^{\#, *}$
}

\begin{abstract}
${ }^{\dagger}$ Center for AIE Research, Shenzhen Key Laboratory of Polymer Science and Technology, Guangdong Research Center for Interfacial Engineering of Functional Materials, College of Material Science and Engineering, Shenzhen University, Shenzhen 518061, P. R. China

${ }^{\S}$ College of Physics and Optoelectronic Engineering, Shenzhen University, Shenzhen 518060, P. R. China

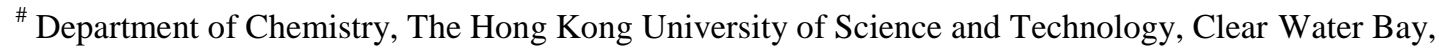
Kowloon, Hong Kong, P. R. China

${ }^{\perp}$ College of Chemistry, Jilin University, 2699 Qianjin Street, Changchun 130012, P. R. China

*E-mail: wangd@szu.edu.cn; ywyang@jlu.edu.cn; tangbenz@ust.hk

Keywords: aggregation-induced emission, Au nanorods, pillararenes, supramolecular materials, synergetic therapy
\end{abstract}

ABSTRACT: Supramolecular approaches have opened up vast possibilities in the construction of versatile functional materials, especially those with stimuli-responsiveness and integrated functionalities of multi-modal diagnosis and synergistic therapeutics. In this study, a hybrid

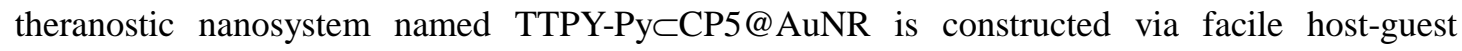
interactions, where TTPY-Py is a photosensitizer with aggregation-induced emission and CP5@AuNR represents the carboxylatopillar[5] arene (CP5)-modified $\mathrm{Au}$ nanorods. TTPY-PyСCP5@AuNR integrates the respective advantages of TTPY-Py and CP5@AuNR such as the high performance of reactive oxygen species (ROS) generation and photothermal conversion, and meanwhile shows fluorescence responses to both temperature and $\mathrm{pH}$ stimuli due to the non-covalent interactions. The successful modification of CP5 macrocycles on AuNRs surfaces can eliminate the cytotoxicity of AuNRs and enable them to serve as the nanocarrier of TTPY-Py for further theranostic application. Significantly, both in vitro and in vivo evaluations 
demonstrate that this supramolecular nanotheranostic system possesses multiple phototheranostic modalities including intensive fluorescence imaging (FLI), photoacoustic imaging (PAI), efficient photodynamic therapy (PDT), and photothermal therapy (PTT), indicating its great potentials for FLI-PAI imaging-guided synergistic PDT-PTT therapy. Besides, TTPY-Py can be released from the nanocarriers upon activating by the acidic environment of lysosomes and then specifically light up mitochondria. This study brings up a new strategy into the design of versatile nanotheranostics for accurate tumor imaging and cancer therapies. 


\section{- INTRODUCTION}

Cancer is one of the major health concerns across the world and tremendous efforts have been made for cancer treatment. The past few decades have witnessed the revolutionary impact of nanotheranostics. In particular, nanomaterials with more reliable diagnostic techniques and enhanced therapeutics have attracted increasing interests. ${ }^{1,2}$ Abundant organic, inorganic, and hybrid nanomaterials with low toxicity, good biocompatibility, and excellent intracellular biostability have been developed through the ingenious integration of different entities. ${ }^{3,4}$ Some nanocontainers, such as the supramolecular or polymeric vesicles, ${ }^{5-7}$ mesoporous silica nanoparticles (MSNs), ${ }^{8}$ and metal-organic frameworks $(\mathrm{MOFs})^{9}$, have been utilized as nanocarriers for drug delivery. ${ }^{10-13}$ In order to achieve excellent tumor suppression and minimal side effects of drugs in cancer therapy, burgeoning non-invasive therapeutic strategies have been developed in recent years, including gene therapy, photothermal therapy (PTT), and photodynamic therapy (PDT). ${ }^{14,15}$ In these therapeutic strategies, various nanomaterials, such as $\mathrm{Au}$ nanoparticles, ${ }^{16} \mathrm{Pt}$ nanoparticles, ${ }^{17}$ upconversion nanoparticles, ${ }^{18} \mathrm{Fe}_{3} \mathrm{O}_{4}$ nanoparticles,,${ }^{19}$ and some typical organic nanomedicines ${ }^{7}$ have been used as effective photothermal agents, photosensitizing agents, and photoacoustic contrast agents. ${ }^{20-22}$ PTT and PDT strategies have minimized the side effects of chemotherapy and operative treatments, but PTT/PDT has obvious defects when used alone. Materials for PTT are always lack of precise imaging-guiding, while photosensitizers for PDT have discounted therapeutic efficacy in hypoxia. ${ }^{7,23}$ Thus, synergistic theranostics, especially those with integrated diagnosis, are in urgent need for cancer therapy. ${ }^{24,25}$ In this context, integrated diagnosis and therapeutics based on nanomaterials are attractive due to their capability of real-time tumor imaging, monitoring the therapeutic process and achieving the maximized 
therapeutic outcomes.

The choice of appropriate diagnostic and therapeutic agents is critically important for the treatment efficacy. On the one hand, the aspect ratio-dependent photothermal effects of $\mathrm{Au}$ nanorods (AuNRs) have been extensively studied owing to their high photothermal conversion efficiency, photoacoustic contrast feature, and great potentials for suppression of tumor. ${ }^{26,27}$ Generally, AuNRs are coated with cetyltrimethylammonium bromide (CTAB) to maintain the colloidal stability because unstable and aggregated AuNRs will lose their unique photothermal property. ${ }^{28}$ However, the surface-coated CTAB possesses very high biotoxicity, which greatly limits the bio-applications of AuNRs. Some other materials, such as biocompatible polymers, mesoporous silica, and small molecules, have also been tried to replace CTAB to enhance the stability of AuNRs. ${ }^{27,29}$ The basic function of coating materials is to maintain the stability and dispersibility of AuNRs. The limitations in some aspects of AuNRs with abovementioned coating materials are the lack of drug/agents loading capacity or the tough methods for preparation. Thus, the property of coating materials is one vital factor that affects the utilization of AuNRs as nanotheranostics. The ligands with carboxyl $(-\mathrm{COOH})$, sulfhydryl $(-\mathrm{SH})$ or amine $\left(-\mathrm{NH}_{2}\right)$ terminals are essential to stabilize Au nanomaterials. On the other hand, photosensitizers with certain diagnostic capacity comes to fluorescence imaging agents. ${ }^{30}$ Other than conventional organic fluorophores with aggregation-caused quenching (ACQ) in the aggregated state, the report of aggregation-induced emission (AIE) concept has led to the revolution in the fields of diagnosis and therapeutics. ${ }^{31-33}$ AIE photosensitizers possess the overwhelming superiority for PDT because the effective restriction of intramolecular motion (RIM) always results in the promotion of not only the ROS generation but also the fluorescence emission, facilitating their real-time monitoring 
of therapeutic process. ${ }^{34-36}$ The elaborated design and tailoring of AIE luminogens (AIEgens) can endow them with the abilities to serve as photosensitizers for PDT. ${ }^{37-39}$ Therefore, nanomaterials based on AIEgens can be prepared not only for accurate tumor-imaging but also for the therapeutic goals.

In terms of the integrating strategies, the development of supramolecular chemistry opens up a new avenue for constructing integrated theranostic nanosystems with well-ordered structures, good biocompatibility, and controllable functions, contributing to their enhanced permeability and retention (EPR) effect, improved pharmacokinetics and optimized drug safety. ${ }^{40-47}$ As a new class of supramolecular synthetic macrocycles, pillar[n]arenes have emerged as a useful class of building blocks in supramolecular nanotheranostics, ${ }^{48-51}$ owing to their unique characteristics, such as rigid pillar-shape structures, facile functionalities, electron-rich cavities, and typical host-guest properties. ${ }^{52-55}$ Appropriate substitutions of the substituent groups at the rims endowed pillar[n]arenes with the ability to incorporate with inorganic nanomaterials, resulting in pillar[n]arene-based supramolecular nanovalves and pillar[n]arene-stabilized Au nanoparticles, CuS nanoparticles, etc. ${ }^{56-58}$ Besides, pillar[n]arenes can be designed to bind with AIE-active photosensitizers containing binding sites via host-guest interactions. ${ }^{59-62}$ For example, Huang, Liu, and co-workers constructed some AIEgen photosensitizers based on the supramolecular complex of water-soluble pillar[5]arene and AIEgen. The reactive oxygen species (ROS) were regulated by changing $\mathrm{pH}$, benefiting from the $\mathrm{pH}-$ responsive host-guest interactions. ${ }^{63}$ 

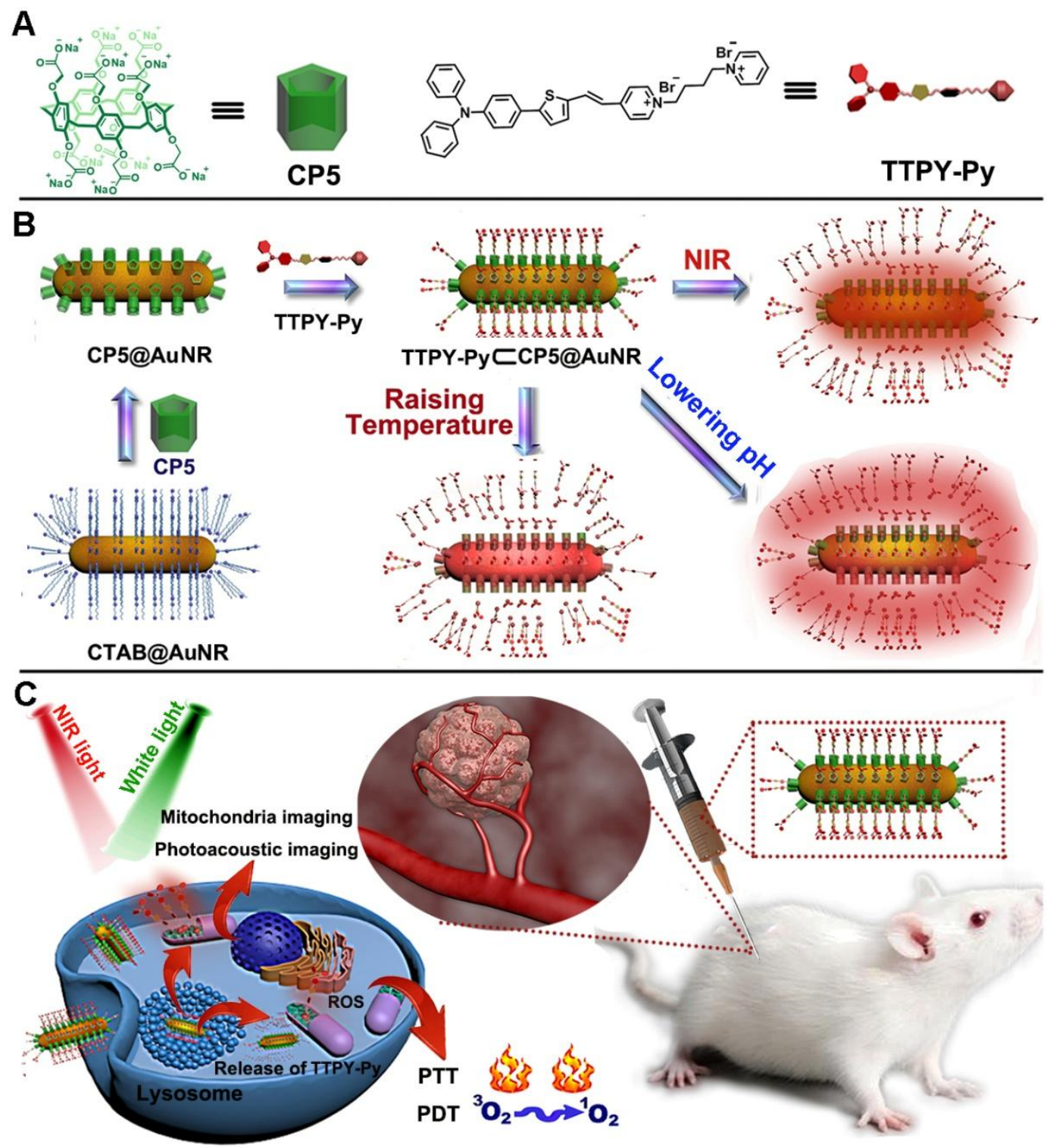

Scheme 1. Schematic illustration for the preparation of the nanomaterials with supramolecular assembly of CP5 and TTPY-Py and their application in synergistic photodynamic-photothermal therapy with real-time tumor-imaging. (A) Chemical structures of

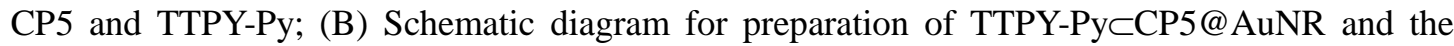
multistimuli-responsive properties; (C) The theranostic applications of the multifunctional materials for FLI-PAI imaging-guided synergistic PDT-PTT therapies (CP5: carboxylatopillar[5]arene; FLI: fluorescene imaging; PAI: photoacoustic imaging; PDT: photodynamic therapy; PTT: photothermal therapy; ROS: reactive oxygen species). 
Inspired by the abovementioned aspects, herein a multifunctional theranostic nanosystem named TTPY-Py $\subset$ CP5@AuNR is constructed (Scheme 1). Different from the previous study which cap macrocycles on the surface of AuNRs via burdensome covalent methods, carboxylatopillar[5]arene (CP5)-modified AuNRs are prepared by a facile ligand-exchange method in this work. The toxicity of AuNRs is successfully avoided and the obtained AuNRs can serve as effective nanocarriers to allow targeting ligand transportation with the aid of CP5. Meanwhile, an AIE-active photosensitizer (TTPY-Py) terminated with pyridinium is synthesized as the guest molecule. CP5@Au could carry TTPY-Py into cancer cells via host-guest interactions between CP5 and TTPY-Py. In this hybrid supramolecular nanomaterial, advantages and functions are combined together: (i) AuNRs stabilized by CP5 possess low cellular toxicity and excellent photothermal effect for PTT and photoacoustic imaging (PAI); (ii) the encapsulation and release of TTPY-Py are controllable under triple stimuli including $\mathrm{pH}$, temperature, and near-infrared (NIR) irradiation; (iii) the escaped TTPY-Py could targetedly turn on mitochondria due to the positive-charged pyridinium terminals and simultaneously generate ROS for PDT. ${ }^{64}$ Thus, the synergistic photothermal-photodynamic theranostics with improved therapeutic effects and imaging-guided treatment are achieved. This work provides a promising strategy to construct new biomedicines and hybrid materials to combine the advantages of advanced techniques with the help of supramolecular chemistry. The imaging signals and therapeutic performance can be manipulated precisely in this way, avoiding the burst drug administration and achieving the non-invasively accurate treatment.

\section{- EXPERIMENTAL SECTION}

2.1 Preparation of materials: CTAB@Au were synthesized with seed-mediated growth. 
CP5@Au were easily prepared by ligand exchange. $6 \mathrm{~mL}$ of CP5 $(1 \mathrm{mM})$ was added to $72 \mathrm{~mL}$ of the solution of quantified CTAB@Au in 2.1. After centrifugation at 7000 rpm for 10 min and the subsequent removal of the supernate, $70 \mathrm{~mL}$ water and another $6 \mathrm{~mL}$ of CP5 $(1 \mathrm{mM})$ was added and fully mixed to maintain the maximum modification. Then the solution was centrifuged again at the same condition to remove the exceeded CP5 ([Au] $=35 \mathrm{ppm}$, quantified by ICP-OES). The above-prepared CP5@Au was diluted to $70 \mathrm{~mL}$ and mixed with $1.2 \mathrm{~mL}$ of TTPY-Py (5 mM). The solution was incubated for $6 \mathrm{~h}$ for sufficient interaction and then the solution was concentrated to $1.5 \mathrm{~mL}$ by centrifugation ( $8000 \mathrm{rpm}, 10 \mathrm{~min}$ ) to obtain the final materials of TTPY-PyСCP5@Au.

2.2. Measurements of the photothermal performance: The water solution of CTAB@Au, CP5@Au, TTPY-Py@Au and TTPY-Py CCP5@Au was continuously exposed to a 808 nm laser at the power of $0.8 \mathrm{~W} / \mathrm{cm}^{2}, 1.0 \mathrm{~W} / \mathrm{cm}^{2}$ and $1.5 \mathrm{~W} / \mathrm{cm}^{2}$ for $10 \mathrm{~min}$, respectively. The corresponding infrared thermal images of the sample tubes were also acquired on a FLIR E6 camera and quantified by FLIR Examiner software. The photothermal properties of TTPY-PyᄃCP5, CP5 and TTPY-Py were also conducted as control. The photothermal conversion efficiency $(\eta)$ of TTPY-Py $\subset \mathrm{CP} 5 @$ Au in aqueous solution was calculated according to the previous literature. ${ }^{65,66}$ The photothermal stability was investigated to repeated irradiation on-off cycles by the $808 \mathrm{~nm}$ laser for $30 \mathrm{~min}$.

2.3 Measurements of the photodynamic performance: DCFH was commonly served as the indicator to investigate the ROS generation efficiency. Firstly, $0.5 \mathrm{~mL}$ DCFH-DA $(1 \mathrm{mM})$ in ethanol was added with $2 \mathrm{~mL} \mathrm{NaOH}$ solution $(10 \mathrm{mM})$ and stirred at room temperature for $30 \mathrm{~min}$, allowing DCFH-DA to be hydrolyzed to DCFH. Then, DCFH was neutralized with $10 \mathrm{~mL}$ PBS $(\mathrm{pH}=7.4)$ and stored in dark. The prepared DCFH solution $(40 \mu \mathrm{M})$ was added into the sample 


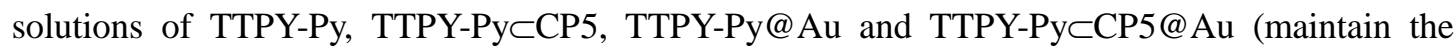
concentration of TTPY-Py to be $1 \mu \mathrm{M}$ in all samples). Subsequently, the mixed solution was irradiated with white light $\left(22.1 \mathrm{~mW} / \mathrm{cm}^{2}\right)$ for different time intervals. The fluorescence intensity of indicator at the emission of $525 \mathrm{~nm}$ was measured on a photoluminescence instrument with the excitation of $488 \mathrm{~nm}$.

\subsection{Intracellular imaging and phototherapeutic study:}

2.4.1 Phototoxicity test: 4T1 cells grown in 1640 culture medium containing $10 \%$ FBS and $1 \%$ antibiotics (penicillin-streptomycin) at $37{ }^{\circ} \mathrm{C}$ in a humidified environment of $5 \% \mathrm{CO}_{2} .4 \mathrm{~T} 1$ cells in a logarithmic growth phase were harvested and seeded in 96-well plates at a density of $5 \times 10^{3}$ cells per well for $24 \mathrm{~h}$ incubation. Then, the cells were added with different concentrations of TTPY-PyCCP5@Au (initial concentration of Au was quantified by ICP-OES) for 12 h incubation. The cells were exposed to white light, $808 \mathrm{~nm}$ laser and white light $+808 \mathrm{~nm}$ laser for $5 \mathrm{~min}$, respectively (power of white light: $22.1 \mathrm{~mW} / \mathrm{cm}^{2}$; power of $808 \mathrm{~nm}$ laser: $0.8 \mathrm{~W} / \mathrm{cm}^{2}$ ). After further incubation for $12 \mathrm{~h}$, the medium was removed and washed with PBS buffer. Fresh serum-free medium containing 10\% CCK-8 was added into above cells and incubated for $2 \mathrm{~h}$ in dark, followed by CCK-8 test.

2.4.2 Intracellular tracking: 4T1 cells were seeded at a suitable density in glass bottom dish and cultured for $24 \mathrm{~h}$, and then incubated with fresh medium containing TTPY-PyᄃCP5@Au ([TTPY-Py $] /[\mathrm{Au}]=0.001 \mu \mathrm{M} / \mu \mathrm{g} ;[$ TTPY-Py $]=2 \mu \mathrm{M} / \mathrm{L} ;[\mathrm{Au}]=2 \mu \mathrm{g} / \mathrm{mL}$ ) for $1 \mathrm{~h}$ and $6 \mathrm{~h}$. After washing with PBS, the cells were costained with Hoechst 33342/LysoTracker Green or Hoechst 33342/MitoTracker Green for 30 min. Bafilomycin A1 (Baf-A1) was added for inhibiting the lysosomal acidity. The irradiation of $808 \mathrm{~nm}$ laser with the power of $0.5 \mathrm{~W} / \mathrm{cm}^{2}$ for $2 \mathrm{~min}$ was 
used to trigger the release of AIEgens. After incubation and treatment, the cells were washed with PBS and imaged by confocal laser scanning microscopy (CLSM) to study the subcellular localization of materials.

2.4.3 Live-dead cell staining: 4T1 cells were seeded and cultured in glass bottom dish for 24

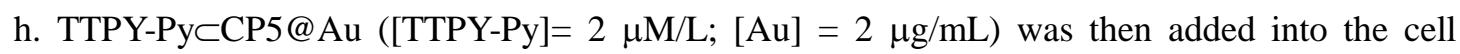
culture medium for $12 \mathrm{~h}$ incubation. After washing and replacing with fresh medium, the cells were irradiated by white light $\left(22.1 \mathrm{~mW} / \mathrm{cm}^{2}\right), 808 \mathrm{~nm}$ laser $\left(0.8 \mathrm{~W} / \mathrm{cm}^{2}\right)$ and white light $+808 \mathrm{~nm}$ laser irradiation for $5 \mathrm{~min}$, respectively, followed by incubation at $37^{\circ} \mathrm{C}$ for another $1 \mathrm{~h}$. Then, the cells were successively stained with PI $(60 \mu \mathrm{g} / \mathrm{mL})$ and FDA $(100 \mu \mathrm{g} / \mathrm{mL})$ in PBS for $10 \mathrm{~min}$. Subsequently, the cells were gently washed and then imaged by CLSM.

\subsection{In vivo imaging and phototherapeuic study:}

2.5.1 In vivo fluorescence, photoacoustic and photothermal imaging: The 4T1 tumor-bearing

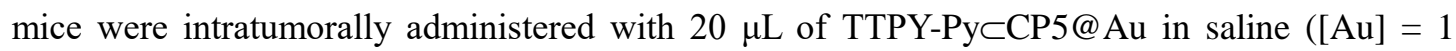
$\mathrm{mg} / \mathrm{mL}$ ) per mouse. Then, at 1, 6, 12 and $24 \mathrm{~h}$ post-injection, the mice were anesthetized using $2 \%$ isoflurane in oxygen and imaged through a Perkinelmer IVIS small-animal fluorescence imaging system. Meanwhile, in vivo photoacoustic imaging was also carried out on a homemade

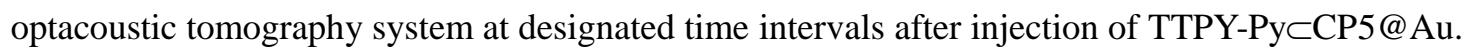
For in vivo photothermal imaging, the infrared thermal images of mice were acquired using an E6 IR camera during the irradiation of $808 \mathrm{~nm}$ laser $\left(0.8 \mathrm{~W} / \mathrm{cm}^{2}\right)$ for $10 \mathrm{~min}$ at $12 \mathrm{~h}$ after administration with TTPY-Py $\subset \mathrm{CP} 5 @$ Au. Mouse injected with saline under the same irradiation condition was used as the control.

2.5.2 In vivo phototherapeutic efficiency: When the inoculated tumor volumes reached about 
$100 \mathrm{~mm}^{3}, 30$ mice were randomly divided into 6 groups, named "Saline", "Saline + white light +

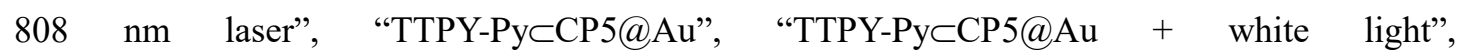

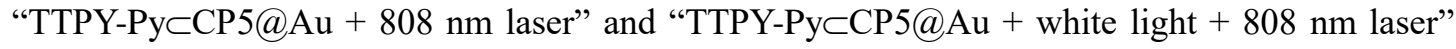
respectively. On day 0, for "Saline" and "TTPY-PyСCP5@Au" groups, $20 \mu \mathrm{L}$ of saline and TTPY-PyсCP5@Au $(1 \mathrm{mg} / \mathrm{mL})$ were intratumorally injected into the 4T1 tumor-bearing mice separately without subsequent light irradiation. In case of “TTPY-PyСCP5@Au + white light”,

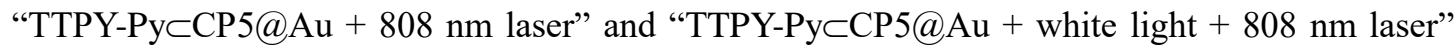
groups, after injection of saline and materials for $12 \mathrm{~h}$, respectively, the tumors of mice in each group were continuously irradiated with white light $\left(22.1 \mathrm{~mW} / \mathrm{cm}^{2}\right)$ and $808 \mathrm{~nm}$ laser $\left(0.8 \mathrm{~W} / \mathrm{cm}^{2}\right)$ for $10 \mathrm{~min}$. After a variety of treatments, the mouse body weight and tumor volume were recorded every 3 days during 15-day study duration. The tumor volume was measured by a vernier caliper and calculated as $\mathrm{V}=a \times b^{2} / 2$. ( $a$ : tumor length; $b$ : tumor width). Relative tumor volume was calculated as $\mathrm{RTV}=\left(\mathrm{V}-\mathrm{V}_{0}\right) / \mathrm{V}_{0}\left(\mathrm{~V}_{0}\right.$ is the initial tumor volume $)$. Relative body weight was calculated as $\mathrm{RBW}=\left(\mathrm{W}-\mathrm{W}_{0}\right) / \mathrm{W}_{0}\left(\mathrm{~W}_{0}\right.$ is the initial mouse body weight $)$. To visualize the antitumor effect, the tumor-bearing mice were photographed on day 0 and 15.

\section{- RESULTES AND DISCUSSION}

\subsection{Synthesis and host-guest interactions}

The AIE-active compound TTPY-Py with the typical binding sites toward the cavity of pillar[5]arene was successfully synthesized through a three-step reaction. As depicted in Figure S1, intermediate compound G1 and G2 was first synthesized by Suzuki-Miyaura coupling reaction and the reaction of 4-methylpyridine and 1,4-dibromobutane, respectively. The subsequent condensation reaction between G1 and G2 produced intermediate compound G3. The covalent 
connection of G3 with pyridine as a binding site finally generated the desired TTPY-Py. Their structures were fully characterized by ${ }^{1} \mathrm{H}$ NMR, ${ }^{13} \mathrm{C}$ NMR, and HRMS (Figure S2 S7). CP5 was synthesized according to our published protocol (Figure S8 S12).
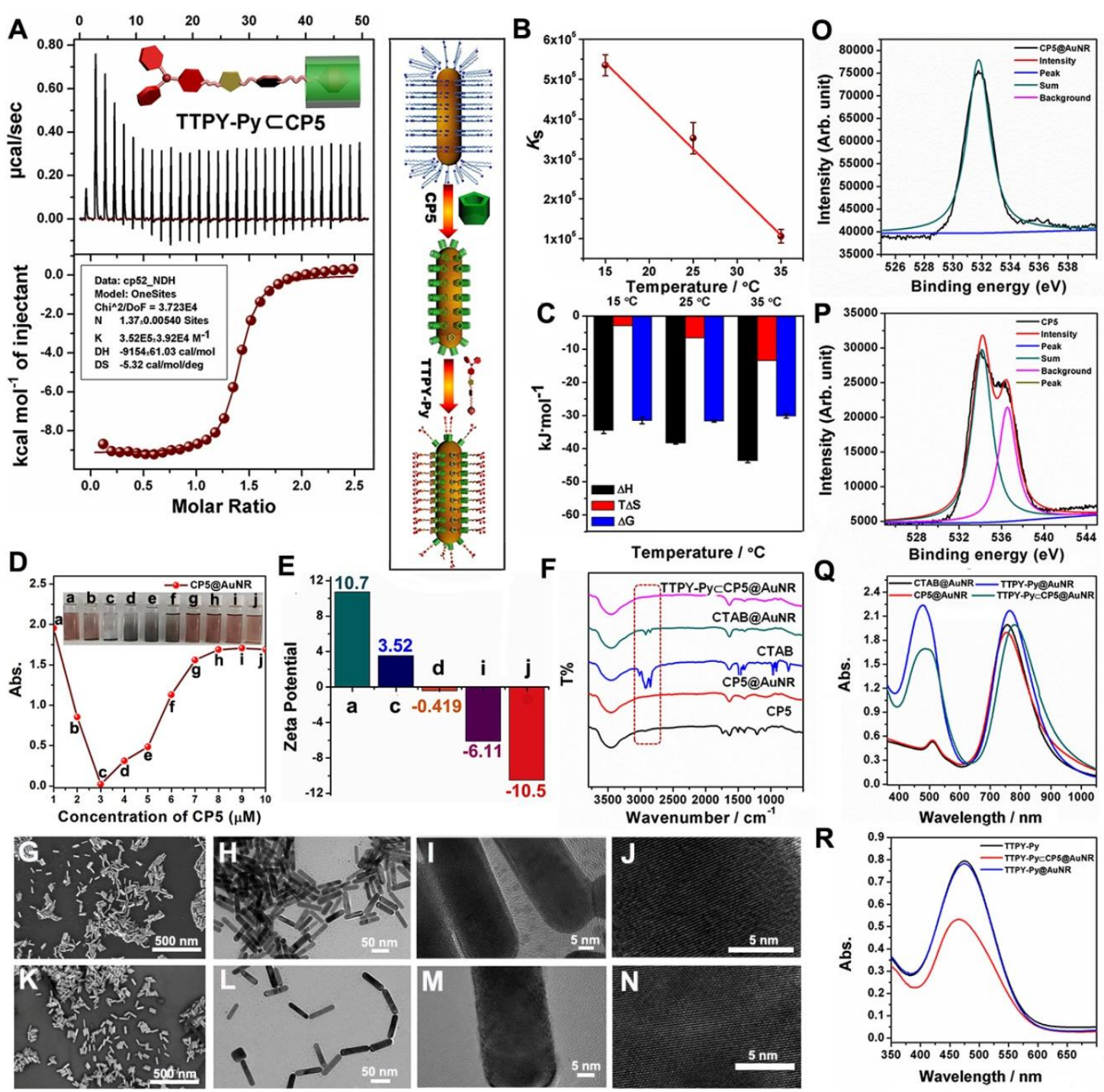

Figure 1. (A) Raw ITC data for 37 sequential injections ( $1 \mu \mathrm{L}$ per injection) of TTPY-Py solution into CP5 solution and net reaction heat obtained by subtracting the dilution heat from the reaction heat, fitted by "one set of binding sites" model (CP5 (1 mM) with TTPY-Py $(8 \mathrm{mM})$ in PBS buffer $(\mathrm{pH}=7.4)$ at $\left.25^{\circ} \mathrm{C}\right)$. (B) Complex stability constant $(K \mathrm{~s})$ for the $1: 1$ inclusion complexation of TTPY-Py $\subset \mathrm{CP} 5$ in PBS buffer $(\mathrm{pH}=7.4)$ at different temperature. (C) Thermodynamic parameters for the 1:1 inclusion complexation. (D) Plots of UV absorbance at $790 \mathrm{~nm}$ for Au nanorods with different concentration of CP5 for ligand change (Insert: corresponding photographs); (E) Zeta potential values of the samples a, c, d, i and j indicated in Figure 1D; (F) FT-IR spectra of 
TTPY-PyᄃCP5@AuNR, CTAB@AuNR, CTAB, CP5@AuNR and CP5. (G) SEM image, (H) TEM image and (I and J) the high resolution of TEM image of CTAB@AuNR. (K) SEM image, (L) TEM image and (M and N) the high resolution of TEM image of CP5@AuNR. XPS spectra of (O) CP5@AuNR and (P) CP5. (Q) Ultraviolet-visible spectra of TTPY-PyᄃCP5@AuNR, CTAB@AuNR, CP5@AuNR and TTPY-Py@AuNR. (R) Loading capacity of CP5@AuNR for TTPY-Py.

The host-guest interaction between CP5 and TTPY-Py was determined by isothermal titration calorimetry (ITC), which is one of the most sensitive methods available for measuring host-guest inclusion complexation. All of the titration data are well fitted by "one set of binding sites" model. The association constants $\left(K_{\mathrm{s}}\right)$ of TTPY-PyсCP5 at $25{ }^{\circ} \mathrm{C}$ was determined to be $(3.52 \pm 0.39) \times$ $10^{5} \mathrm{M}^{-1}$ with the stoichiometry of 1:1 (Figure $1 \mathrm{~A}$ and S13), manifesting the strong binding affinity of TTPY-Py and CP5. In addition, the calorimetric titration of TTPY-PyСCP5 was also conducted at different temperature of $15^{\circ} \mathrm{C}, 25^{\circ} \mathrm{C}$, and $35^{\circ} \mathrm{C}$ (Figure S14 and S15). Elevating temperature significantly weakens the binding affinity as evidenced by the remarkable decrease in $K_{\mathrm{s}}$ values (Table S1 and Figure 1B). ITC experiments also provided thermodynamic parameters for non-covalent inclusion (Figure 1C). The enthalpy and entropic changes govern the complexation free energies. The increased $-T \Delta S^{o}$ caused the decrease in binding stabilities of host-guest systems by rising temperatures. Thus, the rising of temperatures always decreases the binding affinities because of the more unfavorable entropy term $\left(T \Delta S^{\circ}\right)$ accompanied with negative entropic changes $\left(\Delta H^{\mathrm{o}}\right)$

\subsection{Preparation of materials}

Traditional AuNRs, i.e., CTAB@AuNR, with good stability and dispersibility were first prepared by a seed-growth method according to the literature (Figure S16) ${ }^{67}$ The ligand exchange 
process of CTAB@AuNR further generates AuNRs with CP5 capped on surfaces, i.e., CP5@AuNR. As depicted in the UV-Vis spectra (Figure S17), the characteristic lateral and longitudinal absorbance of AuNRs was determined to be at $\sim 510 \mathrm{~nm}$ and $\sim 790 \mathrm{~nm}$, respectively. the AuNRs underwent the process from dispersing to precipitating and then dispersing again due to the changing stability of AuNRs in solution (Figure S17 and Figure 1D) The absorbance at 790 $\mathrm{nm}$ rises first and then drops as the concentration of CP5 increases. This result indicated that upon the gradual addition of $\mathrm{CP} 5$, the AuNRs underwent the process from dispersing to precipitating and then dispersing again due to the changing stability of AuNRs in solution. The addition of a small amount of CP5 was insufficient to stabilize AuNRs, thus resulting in the aggregation and precipitation of Au materials. When enough CP5 was added, the AuNRs was well stabilized with excellent dispersibility. The abovementioned process was also confirmed by SEM images (Figure S18). Besides, the Zeta potential value of CTAB @AuNR (10.7 mV) gradually decreases and then changes from positive $(3.52 \mathrm{mV})$ to negative $(-10.5 \mathrm{mV})$ after modification of CP5 with negatively charged rims (Figure 1E). From the FT-IR spectra, the peak at $2927 \mathrm{~cm}^{-1}$ corresponding to the $\mathrm{C}-\mathrm{H}$ stretching vibration of $\mathrm{CTAB}$ completely disappear after the ligand exchange treatment. All these results suggested the successful surface exchange of CTAB by CP5 (Figure 1F).

As comparison, a typical stabilized ligand toward Au nanoparticles, i.e., sodium citrate, and a rigid compound with similar structure to the monomer of CP5, i.e., $p$-toluenesulfonic acid (TPA), were utilized as controlled and exchanged ligands for stabilization of AuNRs (Figure S19 and S20). Both of the AuNRs precipitated out upon addition of excess sodium citrate and TPA, indicating their ineffectiveness to stabilize AuNRs. Therefore, the capacity of stability and dispersibility of CP5 toward AuNRs can be ascribed to the pillar-shaped and rigid architectures as 
well as the cooperative function of multiple $-\mathrm{COO}^{-}$groups on the rim. The rod-like morphologies of CTAB@AuNR and CP5@AuNR were further observed by SEM and TEM, respectively (Figure 1G-1N). The average length and width of CP5@AuNR were calculated to be $62.98 \mathrm{~nm}$ and 14.17 nm via Nano Measurer analysis (Figure S21). The modification of CP5 on AuNRs were further confirmed by XPS spectra. As shown in Figure 1O, two peaks at $532.9 \mathrm{eV}$ and $536.6 \mathrm{eV}$ were identified for pure $\mathrm{CP}$, ascribing to the carbonyl and hydroxyl oxygen atoms in - $\mathrm{COO}^{-}$moieties of CP5. For CP5@AuNR, only a single peak at $532.1 \mathrm{eV}$ was identified at the O1s spectra region (Figure 1P). The assimilating or identical of the electronic environments of two kinds of $\mathrm{O}$ atoms in $-\mathrm{COO}^{-}$is attributed to the interaction of AuNRs' surfaces to the carboxylate groups. The XPS spectra for Au 4f photoelectrons of CTAB@AuNR and CP5@AuNR were very similar (Figure S22), suggesting that no degradation occurred in AuNRs. The elemental distributions of CTAB@AuNR and CP5@AuNR were also observed by EDS mapping (Figure S23).

Subsequently, AIE-active TTPY-Py molecules were hybridized onto the surface of CP5@AuNR with the aid of the CP5 cavity through host-guest interactions. As shown in Figure 1Q, the UV-Vis spectra of CTAB@AuNR and CP5@AuNR were identical due to the changeless structure of AuNRs, while the absorbance at $475-500 \mathrm{~nm}$ increased due to the addition of TTPY-Py and the peak at $\sim 790 \mathrm{~nm}$ underwent slight red-shift after loading with TTPY-Py to construct TTPY-PyСCP5@AuNR. For comparison, the same concentration of TTPY-Py was also added into CTAB@AuNR. In this case, the absorbance at 475-500 nm showed more increase without shift at $\sim 790 \mathrm{~nm}$, because there was no interaction between TTPY-Py and CTAB@AuNR. Both of the less increase at $475 \sim 500 \mathrm{~nm}$ and red-shift at $\sim 790 \mathrm{~nm}$ indicated the interactions between TTPY-Py and CP5@AuNR. Furthermore, in order to investigate the encapsulation 
capacity, the UV-Vis absorbances of residual TTPY-Py in the supernates of pure TTPY-Py, TTPY-PyСCP5@AuNR, and TTPY-Py@AuNR after sufficient centrifugation were measured. The reduced TTPY-Py in supernate was loaded in CP5@AuNR, manifesting the ability of CP5@AuNR to serve as nanocarriers. (Figure 1R, Figure S24, S25 and Table S2). Upon the increase in the feed content of TTPY-Py, the encapsulation efficiencies were decreased due to the limited amounts of CP5 on the surfaces of AuNRs. The maximum capacity of CP5@AuNR to load TTPY-Py reached $\sim 0.001 \mu \mathrm{M} / \mu \mathrm{g}$ ([TTPY-Py]/[AuNR]).

\subsection{Optical properties and stimuli-responsiveness}

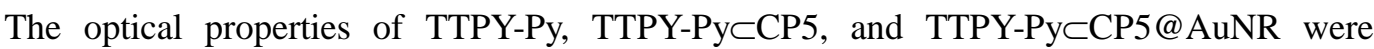
investigated by their UV-Vis absorbance and fluorescence emission. Upon the gradual addition of unfavorable solvent, i.e., THF, into the $\mathrm{MeOH}$ solution of TTPY-Py, the fluorescence intensity at $680 \mathrm{~nm}$ was enhanced continuously (Figure S26). This demonstrated that TTPY-Py possesses typical AIE characteristics due to the restriction of intramolecular rotations (RIR) in aggregate states. Significantly, CP5 possessed the similar ability to limit the intramolecular rotations by encircling on the terminal binding site of TTPY-Py via host-guest interaction. Therefore, the fluorescence intensity also gradually increased upon addition of CP5 and reached to the plateau when they were at the same concentration for sufficient complex. This result was consistent with the stoichiometry of TTPY-Py $\subset$ CP5 at 1:1. The emission also underwent $\sim 20 \mathrm{~nm}$ red-shift at 700

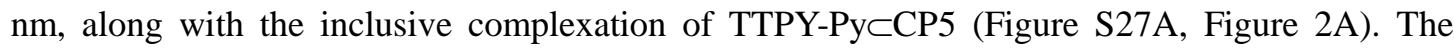
host-guest interaction between TTPY-Py and CP5 was further demonstrated in UV-Vis spectra. The absorbance of TTPY-PyСCP5 at $475 \mathrm{~nm}$ decreased slightly until the molar ratio reached 1:1

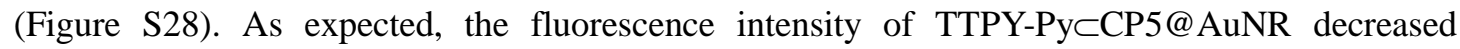


remarkably due to the overlapped longitudinal absorbance of CP5@AuNR at 790 nm and fluorescence emission of TTPY-Py $\subset$ CP5 at $700 \mathrm{~nm}$. However, it is still stronger than the fluorescence of TTPY-Py@Au due to the protective screen of CP5 (Figure S27B).
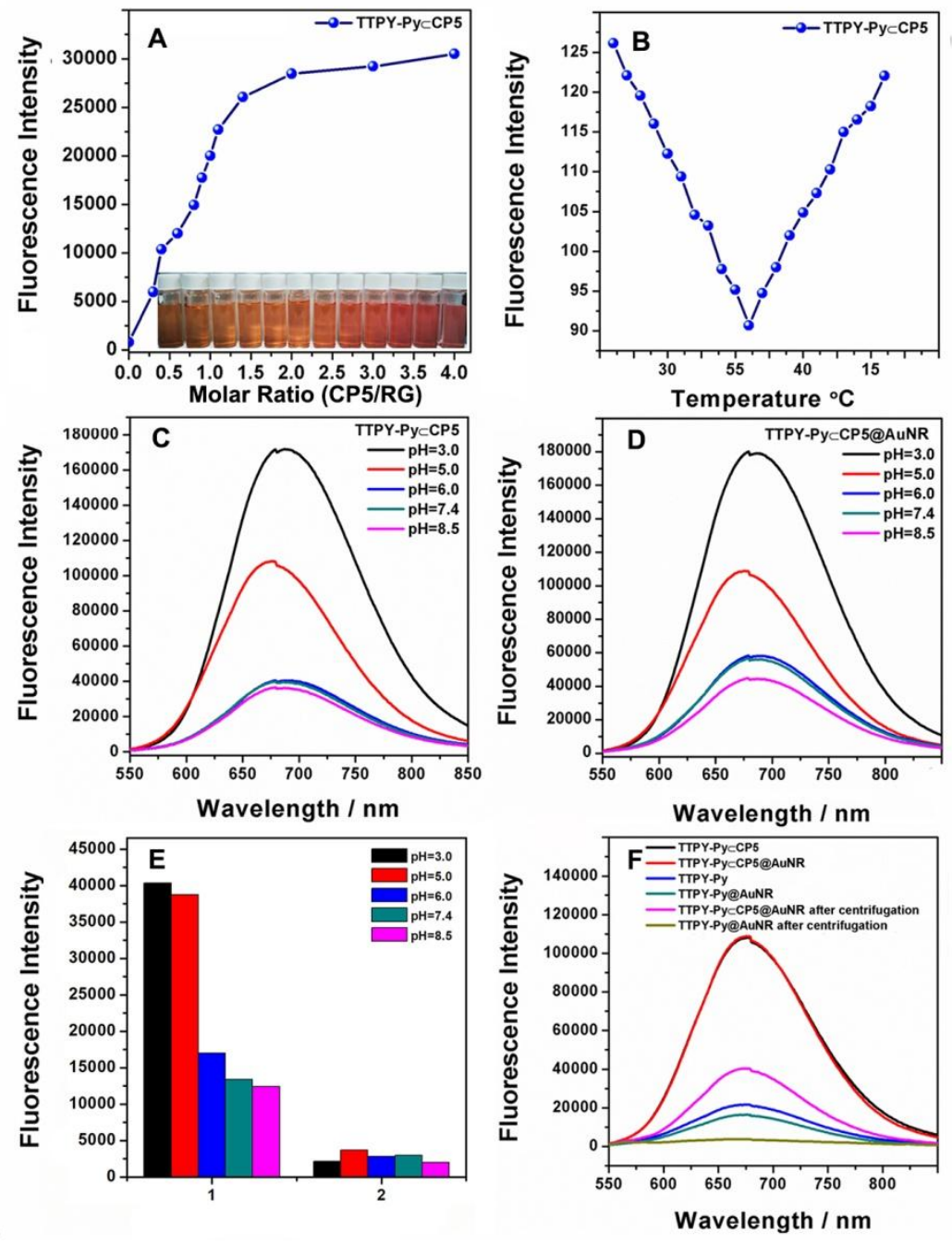

Figure 2. (A) Plots of fluorescence emission of TTPY-Py upon gradual addition of CP5 (Insert: photographs of samples upon addition of CP5 into TTPY-Py). (B) Changes of fluorescence emission of TTPY-PyᄃCP5 upon changing temperature. Fluorescence emission of (C)

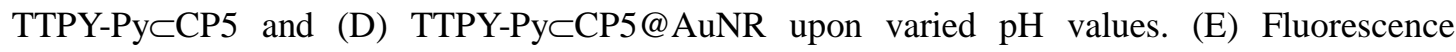
emission of materials after centrifugation (1: TTPY-PyСCP5@AuNR; 2: TTPY-Py@AuNR). (F) Fluorescence emission of different samples at the same $\mathrm{pH}$ value of 5.0. 
One of the crucial features of non-covalent interactions is their stimuli-responsiveness, especially in the process of host-guest recognition. Similarly, in the supramolecular inclusion of TTPY-Py $\subset$ CP5, elevated temperatures result in the decrease of fluorescence intensity (Figure 2B). This observation can be explained by the reduced stability and binding affinity of host-guest systems due to the raising complexation free energy governed by the more unfavorable entropy

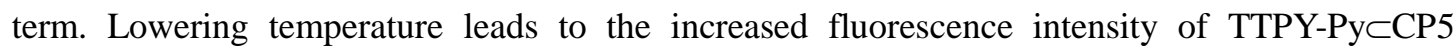
because of the strengthened host-guest interaction. As comparison, the fluorescence intensity of pure TTPY-Py, TTPY-PyСCP5@AuNR and TTPY-Py@AuNR showed negligible fluorescence changes upon variation of temperature (Figure S29 and S30) owing to their very weak fluorescence. The fluorescence of TTPY-PyСCP5@Au and TTPY-Py@Au were mainly quenched by the longitudinal plasma resonance of AuNRs.

Besides temperature, the fluorescence intensity of TTPY-PyᄃCP5 can also be regulated by varying $\mathrm{pH}$ values. As shown in Figure $2 \mathrm{C}$, the fluorescence intensity remarkably increases upon lowering the $\mathrm{pH}$, because the $-\mathrm{COO}^{-}$groups on the rims of $\mathrm{CP} 5$ can change into $-\mathrm{COOH}$ in an acidic environment to induce the less water solubility of CP5 and the dissociation of TTPY-Py from the cavity of CP5. Similar results were also observed with TTPY-PyᄃCP5@AuNR (Figure 2D). Although the host-guest inclusion was weakened in lowered $\mathrm{pH}$, the increased fluorescence intensity was dominantly influenced by the poorer solubility. Moreover, TTPY-PyᄃCP5@AuNR after centrifugation exhibited similar variation tendency and remarkably stronger fluorescence intensity compared with the weakly fluorescent TTPY-Py@ AuNR (Figure 2E, Figure S31). These results further confirmed the loading of TTPY-Py in CP5@AuNR and the pH-responsive property

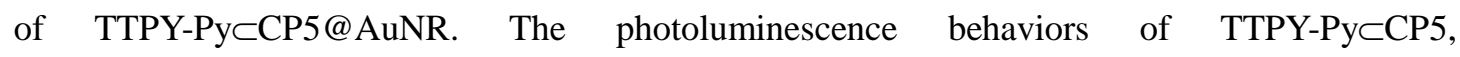


TTPY-PyᄃCP5@AuNR, pure TTPY-Py, and TTPY-Py@AuNR were compared at the same pathological condition of $\mathrm{pH} 5$ and the normal condition of $\mathrm{pH}$ 7.4. As shown in Figure $2 \mathrm{~F}$ and Figure S32A, TTPY-Py $\subset$ CP5 and TTPY-Py $\subset$ CP5@AuNR showed the strongest fluorescence intensity. The influence of the solubility was displayed in Figure S32B.
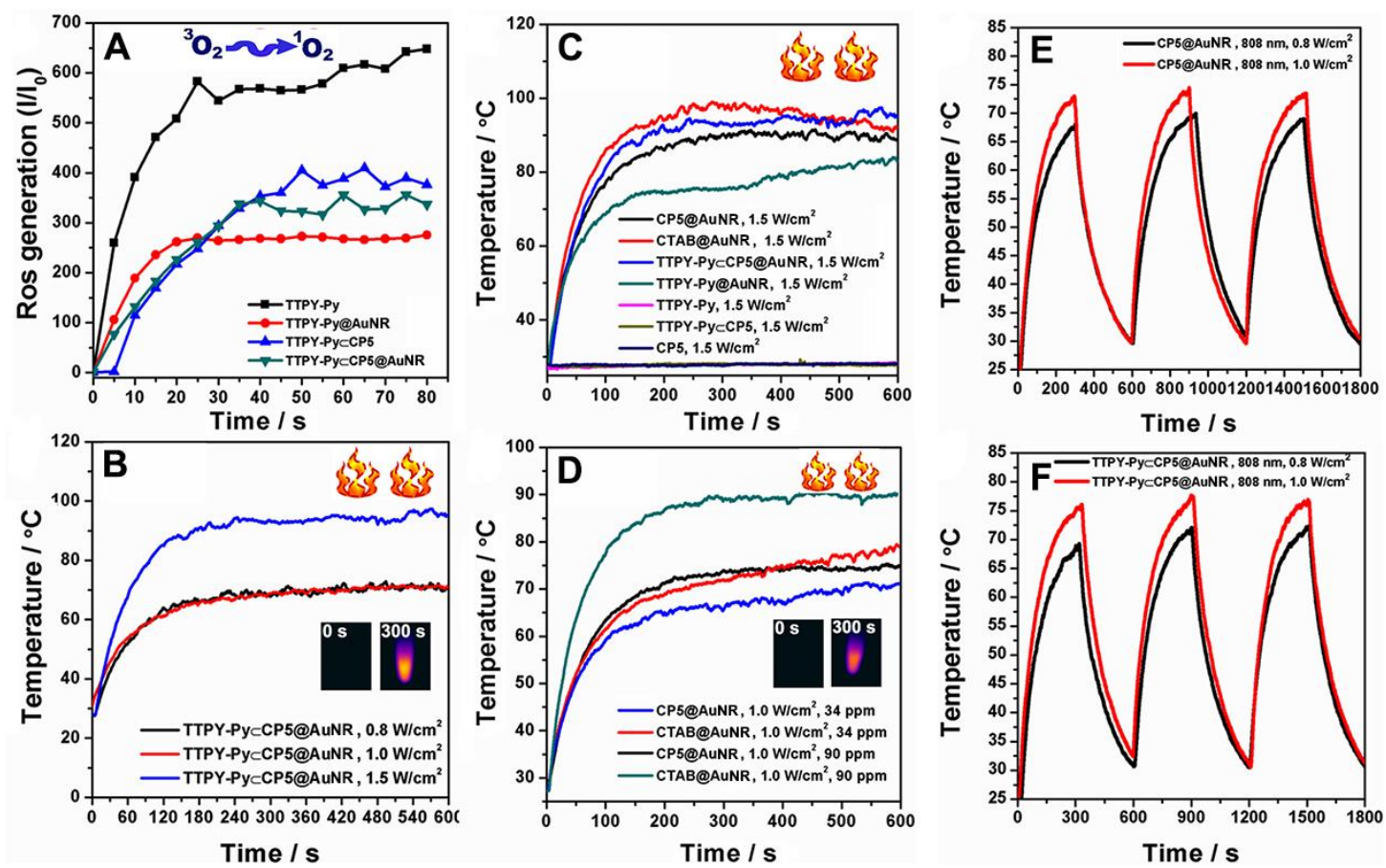

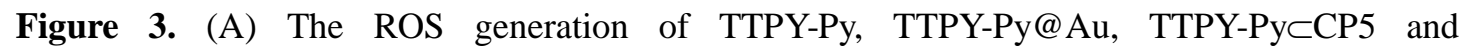
TTPY-Py $\subset$ CP5@AuNR with the DCFH-DA as an indicator. (B) Photothermal property of TTPY-Py $\subset$ CP5@AuNR upon different power of $808 \mathrm{~nm}$ laser irradiation. (C) Photothermal property of TTPY-PyсCP5@AuNR, CTAB@AuNR, CP5@AuNR, TTPY-Py@AuNR, TTPY-Py $\subset$ CP5, CP5 and TTPY-Py. (D) Photothermal property of CP5@AuNR and CTAB@AuNR with different concentration of Au. Thermal cycle of (E) CP5@AuNR and (F) TTPY-PyᄃCP5@AuNR upon irradiation of 808 nm laser. 


\subsection{Photodynamic and photothermal performance}

One of the most significant factors for PDT is the ROS generation performance of photosensitizers, since cell apoptosis can be induced by ROS. Generally speaking, molecules with donor-acceptor (D-A) structure tend to promote the ROS generation/Many AIEgens with donor-acceptor (D-A) structure have been reported to show high ROS generation. ${ }^{68}$ Thus we speculated that D-A structured TTPY-Py would efficiently generate ROS to achieve the goal of PDT. To verify our hypothesis, the ROS generation efficiency of TTPY-Py, TTPY-Py@AuNR,

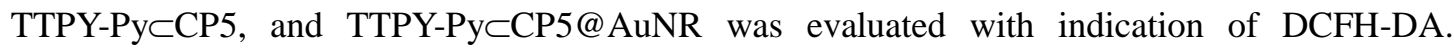
DCFH-DA is a commercially available indicator which can show green fluorescence upon irreversible reaction with ROS. As illustrated in Figure S33 and 3A, a rapid enhancement of fluorescence intensity of DCFH at $525 \mathrm{~nm}$ was observed upon the white light irradiation in the

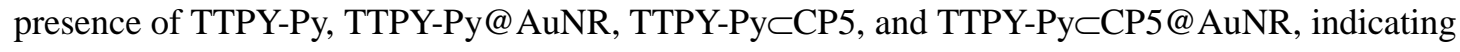
the high ROS generation performance of TTPY-Py and its complexes. In the presence of pure TTPY-Py, the fluorescence intensity of DCFH raised over 600-fold after 1 min irradiation of white light. However, the fluorescence intensity reached over 350-fold after 1 min irradiation by

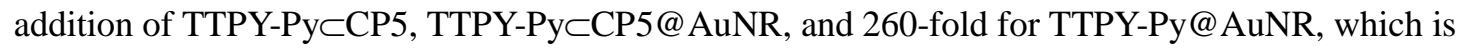
much lower than that of pure TTPY-Py. These experimental results demonstrated that the pure TTPY-Py exhibited superior ROS generation efficiency compared with the host-guest complex or the hybrid materials, which might be attributed to the promoted intersystem crossing decay and stronger D-A interaction.

Benefiting from the rod-like shape and typical surface electron resonance of AuNRs, TTPY-PyСCP5@AuNR exhibited excellent photothermal performance. As shown in Figure 3B, 


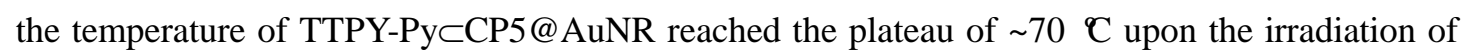
$808 \mathrm{~nm}$ laser with the power of $0.8 \mathrm{~W} / \mathrm{cm}^{2}$ and $1.0 \mathrm{~W} / \mathrm{cm}^{2}$, while the temperature reached by $97{ }^{\circ} \mathrm{C}$ within $2 \mathrm{~min}$ with the laser power of $1.5 \mathrm{~W} / \mathrm{cm}^{2}$. Meanwhile, it is worthy mentioning that both CP5-modified AuNRs and TTPY-PyᄃCP5@AuNR have negligible influence on the photothermal efficiency of AuNRs (Figure 3C, Figure S34 and Figure S35), but TTPY-Py-modified AuNRs showed less increase in temperature than naked AuNRs. This result indicated that TTPY-Py inserted a negative effect on the photothermal performance of AuNRs without the assistance of CP5. As depicted in Figure 3D, the photothermal properties of both CP5@AuNR and CTAB@AuNR depend on the concentration, and the use of high concentration will definitely improve the photothermal conversion efficiency. Furthermore, no obvious changes in temperature were observed for CP5@AuNR and TTPY-Py $\subset$ CP5@AuNR during the cycling process after irradiation with $808 \mathrm{~nm}$ laser for three cycles, indicating their satisfactory photostability (Figure 3E and 3F). In summary, TTPY-PyСCP5@AuNR exhibited high photothermal performance with concentration- and temperature-dependent characteristics as well as excellent photostability. These properties would contribute to their great potentials for PTT application in biological systems. 

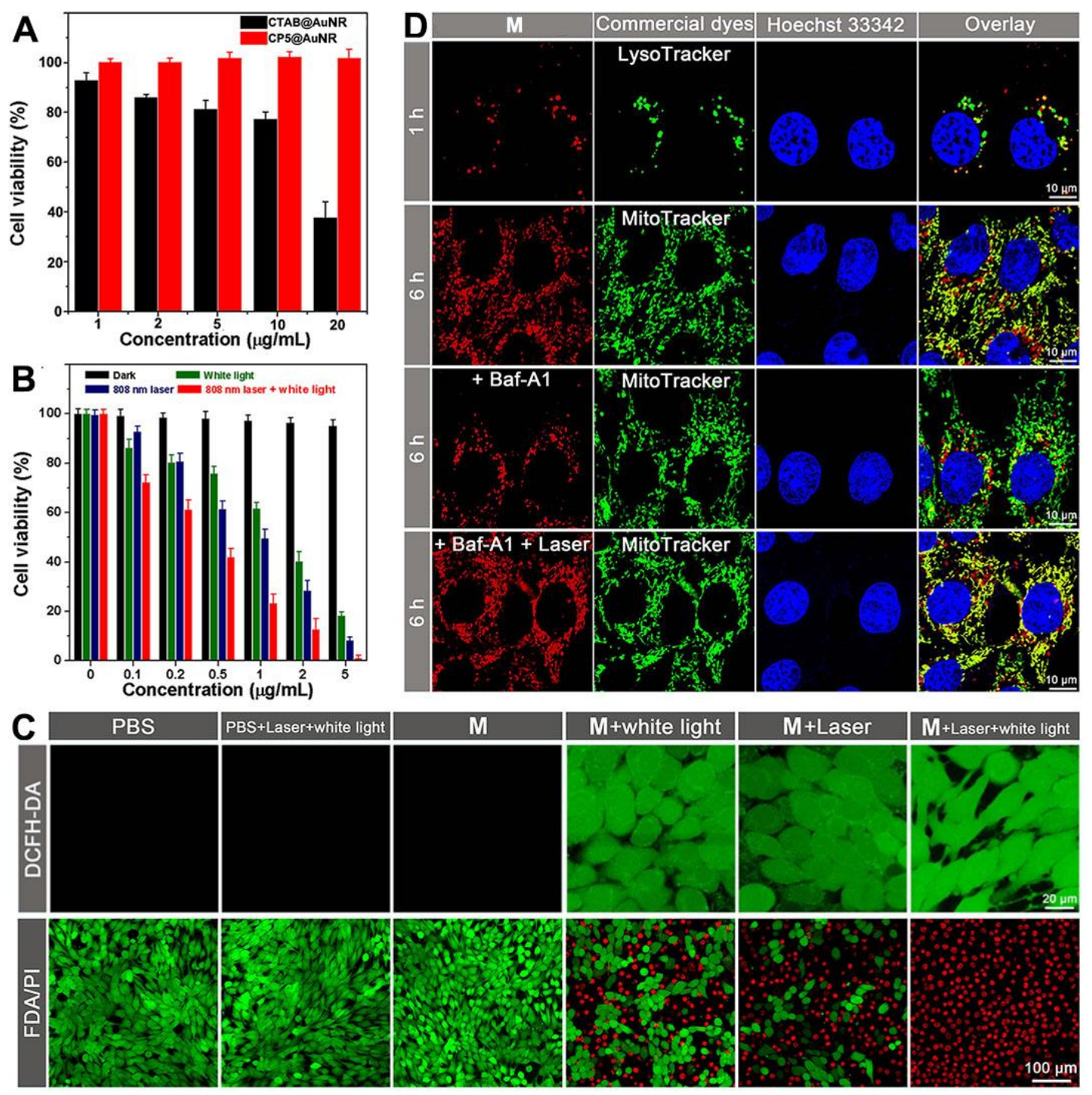

Figure 4. (A) Cell viabilities of 4T1 cells after incubation with CTAB@AuNR or CP5@AuNR for $24 \mathrm{~h}$ in the dark determined by CCK-8 assay (mean $\pm \mathrm{SD}, \mathrm{n}=6$ ). (B) Cell viabilities of $4 \mathrm{~T} 1$ cells

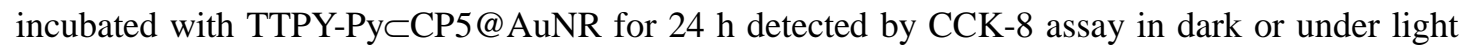
irradiation condition (mean $\pm \mathrm{SD}, \mathrm{n}=6)$. The white light $\left(22.1 \mathrm{~mW} / \mathrm{cm}^{2}\right)$ and/or $808 \mathrm{~nm}$ laser $(0.8$ $\mathrm{W} / \mathrm{cm}^{2}$ ) were performed at $12 \mathrm{~h}$ post incubation for $5 \mathrm{~min}$. (C) Upper: Intracellular total ROS activity assay: 4T1 cells incubated with TTPY-PyᄃCP5@AuNR for 12 h, added with DCFH-DA and treated with DCFH-DA. After irradiation, the cells were incubated for another $12 \mathrm{~h}$; Bottom: Live/dead cell staining of 4T1 cells after treatments with various formulations with or without light irradiation. The concentration of TTPY-PyᄃCP5@AuNR was unified to be $2 \mu \mathrm{g} / \mathrm{mL}$. (D)

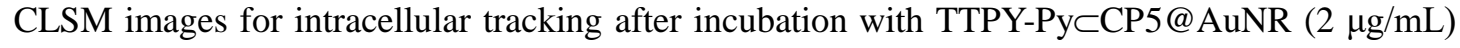
for different time and treatments with various conditions by using commercial multiple fluorescent

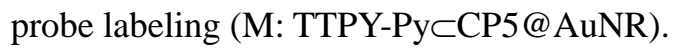


3.5 Cellular imaging and therapeutic studies

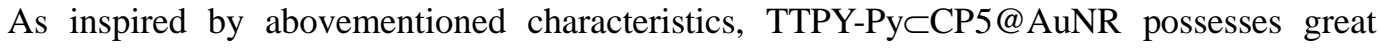
promise for integrated phototherapeutics and multimode diagnosis. Thus we evaluated the in vitro synergistic phototheranostic effficacy of TTPY-PyᄃCP5@AuNR against mouse breast cancer 4T1 cells. First of all, the cytotoxicity of materials in dark was estimated by CCK-8 assay. The results shown in Figure 4A demonstrated that CP5@AuNR had no obvious toxicity to 4T1 cells compared with CTAB@AuNR, even at a relative high concentration. This observation indicated that the modification of CP5 on the surface of AuNRs efficiently dislodged toxic CTAB and

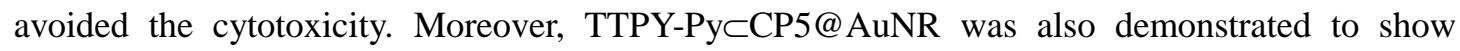
excellent biocompatibility (Figure S36). Subsequently, the white light- and NIR laser-induced tumoricidal effects of TTPY-PyСCP5@AuNR were investigated by CCK-8 assay. As illustrated in Figure 4B, TTPY-PyСCP5@AuNR exhibited obvious phototoxicity toward 4T1 cells upon the irradiation of white light $\left(22.1 \mathrm{~mW} / \mathrm{cm}^{2}, 5 \mathrm{~min}\right)$ or NIR laser $\left(808 \mathrm{~nm}, 0.8 \mathrm{~W} / \mathrm{cm}^{2}, 5 \mathrm{~min}\right)$. Typically, upon the cooperated irradiation of white light and NIR laser, TTPY-PyᄃCP5@AuNR possessed ultra-high tumoricidal efficacy even at a very low concentration, indicating that TTPY-Py $\subset$ CP5@AuNR exhibited efficient synergistic phototheranostics toward 4T1 cells.

Furthermore, the efficacy of intracellular ROS generation was investigated by DCFH-DA in 4T1 cells upon irradiation. As depicted in Figure 4C and S37, 4T1 cells treated with TTPY-PyCCP5@AuNR and DCFH-DA exhibited strong green fluorescence upon white light and NIR irradiation, while no fluorescence was detected in controlled PBS. These results demonstrated that TTPY-Py $\subset$ CP5@AuNR can efficiently generate ROS to show excellent phototoxicity against

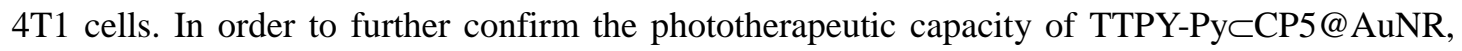


fluorescein diacetate/propidium iodide (FDA/PI) double staining method was performed, in which live and dead 4T1 cells were distinguished by green and red colors, respectively (Figure 4C and S38). As expected, strong red signals were observed when the cells were treated with TTPY-Py $\subset$ CP5@AuNR and irradiated solely by white light or NIR laser. When cooperated

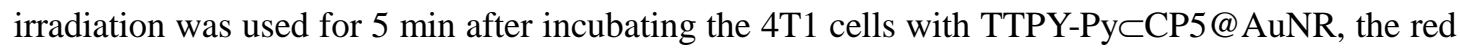
signals can be observed in almost the whole regions, which was indicative of the efficient phototoxicity of TTPY-Py $\subset$ CP5@AuNR as synergistic nonatheranostics. By contrast, no red fluorescence signals but strong green fluorescence were detected in the controlled groups of PBS

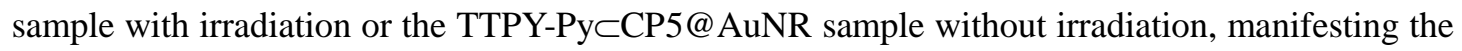
desirable biocompatibility and the negligible side effect of same laser.

Next, the intracellular fate and cell staining capability of TTPY-PyᄃCP5@AuNR were examined using a confocal laser scanning microscope. As depicted in Figure 4D, the TTPY-Py molecules carried in TTPY-PyᄃCP5@AuNR can be internalized into cells within 1h incubation and firstly entrapped into lysosomes. After $6 \mathrm{~h}$ incubation, the acidic environment of lysosomes $(\mathrm{pH}=5.0 \sim 5.5)$ triggered the release of TTPY-Py through weakening the host-guest interaction, which then specifically lighted up the mitochondria region as indicated by the co-staining results with a commercial mitochondrion imaging agent (MitoTracker). The mitochondria-targeting capacity of TTPY-Py might be ascribed to its terminally positive-charged pyridinium group. Notably, individual TTPY-Py without the transportation of CP5@AuNR carriers cannot reach mitochondria but only located within lysosomes (Figure S39). This result clearly verified the significance of supramolecular nanocarriers. In order to confirm the stimuli-responsiveness in lysosomes, Baf-A1 was then used to inhibit the acidity and lysosomal degradation to block 
TTPY-Py within lysosomes. In this case, less overlapped fluorescence was observed in $4 \mathrm{~T} 1$ cells, suggesting the importance of acidic lysosomal microenvironment. In addition, the raised temperature of TTPY-Py $\subset$ CP5@AuNR caused by $808 \mathrm{~nm}$ laser irradiation could also activate the release of TTPY-Py from Au NR-based nanocarriers to achieve the lighting up of mitochondria. Therefore, the outstanding performance in targeted mitochondria imaging of TTPY-PyᄃCP5@AuNR is supposed to benefit from the acidic- and temperature-triggered delivery of TTPY-Py due to the change of host-guest interactions.

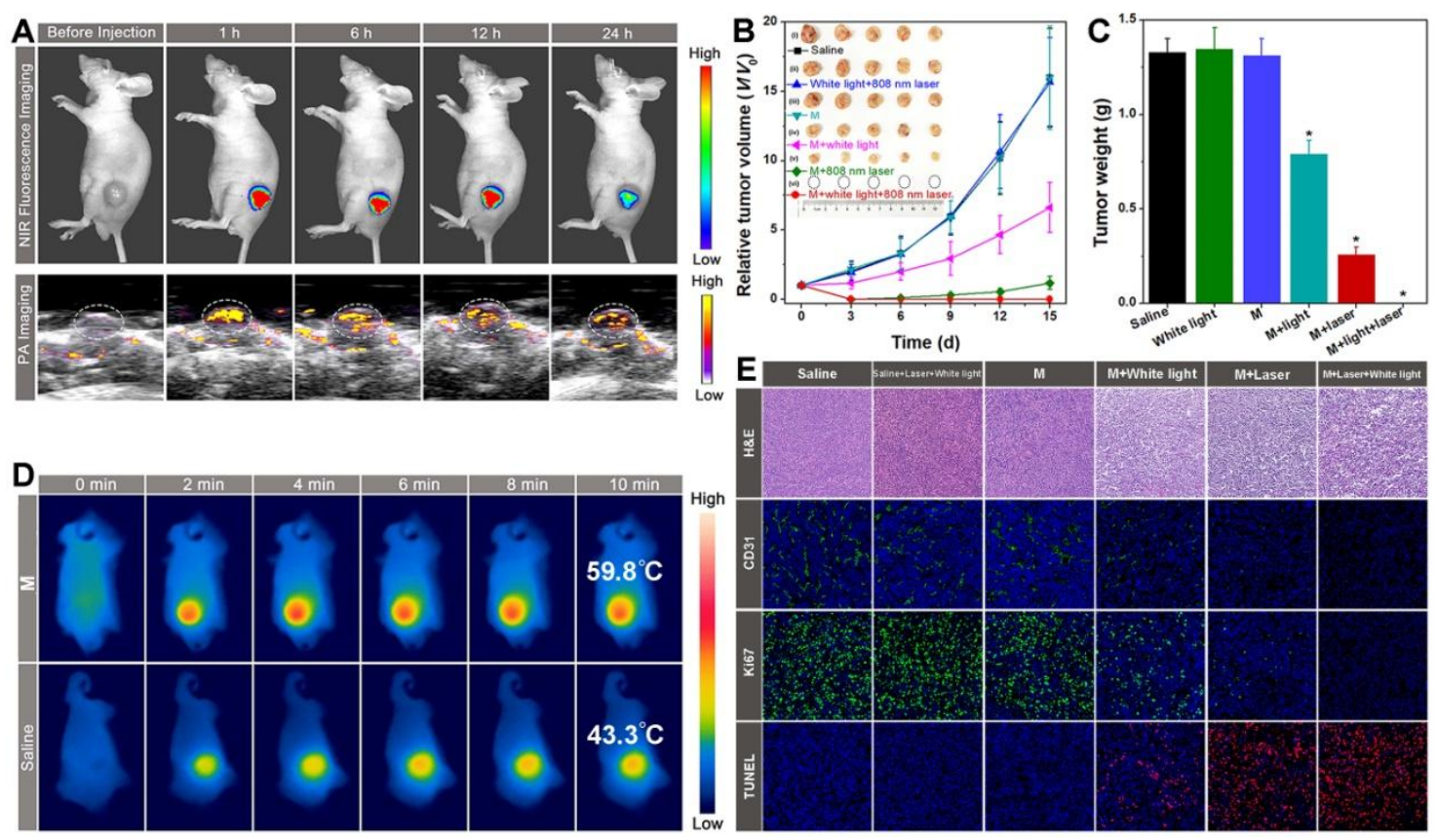

Figure 5. (A) In vivo FLI (upper row) and PAI of tumor tissues (lower row) of tumor-bearing mice at different monitoring times after intratumoral injection of TTPY-PyᄃCP5@AuNR. (B) Infrared thermal images of $4 \mathrm{~T} 1$ tumor-bearing mice under $808 \mathrm{~nm}$ laser irradiation $(0.8 \mathrm{~W} / \mathrm{cm} 2)$ for different times after injection of TTPY-PyСCP5@AuNR. (C) (C) Tumor volumes and (D) tumor weights of $4 \mathrm{~T} 1$ tumor-bearing mice treated with saline, white light+808 nm laser,

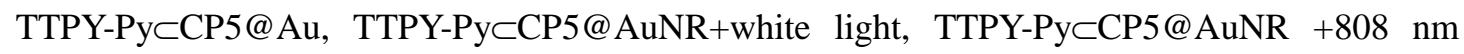
laser and TTPY-Py $\subset$ CP5@AuNR+white light+808 nm laser (insert: Photographs of tumors on the 15th day after different treatments (i: saline; ii: white light+808 $\mathrm{nm}$ laser; iii:

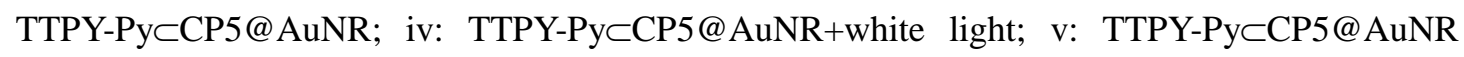
+808 nm laser; vi: TTPY-Py CP5@AuNR+white light+808 nm laser.) ). (E) H\&E, CD31, Ki67 
and TUNEL staining analysis of tumor tissues after treatments of saline, saline with 808 laser and white irradiation, M (TTPY-PyᄃCP5@AuNR), M with white light irradiation, M with 808 laser irradiation and M with 808 laser and white irradiation, respectively. Ki67 stained proliferative cells with green color, CD31 stained neovessels with green color and TUNEL-positive cells were stained red.

3.6 In vivo imaging and therapeutic studies:

To satisfy the ultimate goal of efficient nanotheranostics for the accurate and integrated diagnosis and therapeutics, in vivo imaging and tumoricidal performance were investigated by injecting the nanomaterials into tumors of $4 \mathrm{~T} 1$ tumor-bearing mice. The fluorescence imaging (FLI) and photoacoustic imaging (PAI) results illustrated in Figure 5A demonstrated that TTPY-PyCCP5@AuNR possess excellent capacity of FLI and PAI, benefiting from the AIE-active TTPY-Py and photothermal AuNRs, respectively. Both fluorescent and photoacoustic signals were intensively observed in tumor regions after $1 \mathrm{~h}$ injection and sustained for about $12 \mathrm{~h}$, indicating the prominent accumulation of TTPY-Py $\subset \mathrm{CP} 5 @$ AuNR in tumors. The integrating FLI and PAI capacities endowed TTPY-PyСCP5@AuNR with great potential for accurate tumor recognition and imaging-monitored therapy.

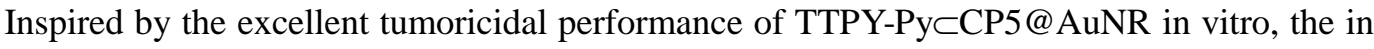
vivo phototheranostic performance and antitumor efficacy in the $4 \mathrm{~T} 1$ tumor-bearing mice were conducted. As indicated by the photographs of tumors and the profile of relative tumor volumes shown in Figure 5B, TTPY-Py $\subset$ CP5@AuNR exhibited synergistic photothermal and photodynamic effects towards tumors upon the irradiation of both white light and NIR laser. The tumors were partially inhibited and eliminated upon the alone irradiation of white light or NIR laser, while the tumor growth was completely inhibited by $100 \%$ without any recurrence upon the 
synergistic PTT-PDT (Figure S40). This conclusion was further confirmed by the tumor weights and tumor growth inhibition (Figure 5C and S41). In sharp contrast, the tumors in the controlled groups (sample treated with saline, irradiation without TTPY-Py $\subset$ CP5@AuNR, and with TTPY-Py $\subset$ CP5@AuNR but without irradiation) grew rapidly during 15 days, indicating that the phototheranostic capacity could not be activated in the absence of either TTPY-PyᄃCP5@AuNR or irradiation. To visually investigate the photothermal performance in vivo, the temperature changes of tumor were monitored in real time with the help of an infrared thermal camera. As shown in Figure 5D and Figure S42, regional hyperthermia was generated and observed in tumor within $2 \mathrm{~min}$, and the temperature reached to around $60{ }^{\circ} \mathrm{C}$ after irradiation using a NIR laser for 5 min, demonstrated the great photothermal effect of TTPY-PyᄃCP5@AuNR. In comparison, the temperature in tumor had slight change when the tumor-bearing mice was administrated with saline and NIR laser, suggesting the negligible laser-induced damage toward bodies. Additionally, there was no significant difference in body weights of mice no matter whether the mice were

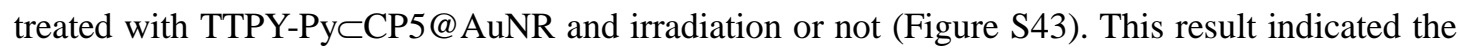
importance of the supramolecular nanocarriers and the almost negligible side effect of the as-prepared nanotheranostics on bodies.

Subsequently, histological and immunohistochemical studies were conducted for further evaluation of side effects and the analyses of the in vivo therapeutic mechanism. As illustrated in Figure 5E, hematoxylin and eosin (H\&E) staining and the terminal deoxynucleotidyl transferase dUTP nick end labeling (TUNEL) immunofluorescence staining of tumor tissue slices showed conspicuous karyopyknosis of tumor cells and sever cell apoptosis, when they were administrated with TTPY-Py $\subset$ CP5@AuNR and irradiations. This observation revealed the effective destruction 
of tumors which was caused by synergistic hyperthermia and ROS of materials upon irradiation with white light and NIR laser. Besides, the platelet endothelial cell adhesion molecule-1 (CD31)-positive vessel formation proved the inhibition of tumor proliferation by the invisible new microvessels. The efficient inhibition of cell proliferation was then confirmed by the Ki67 staining. By contrast, few apoptotic cells, abundant cell proliferation, and massive new microvessels were observed in controlled groups. All these results indicated that the TTPY-PyᄃCP5@AuNR system together with the cooperative irradiation of white light and NIR laser exhibited efficient capacity for synergistic PTT and PDT. After treatment for 15 days, H\&E-stained slice images of major organs (Figure S44), the blood biochemistry analysis (Table S3), and the liver and renal function analysis (Table S4) were conducted and compared respectively. The obtained results demonstrated that there was no obvious inflammatory damage in major organs and the supramolecular nanomaterials carried with TTPY-Py can serve as promise nanotheranostics with excellent biocompatibility.

\section{- CONCLUSION}

In summary, we successfully developed a new AIE-active theranostic nanosystem, i.e., TTPY-Py $\subset$ CP5@AuNR, via a facile supramolecular strategy, which exhibited multiple functions to integrate FLI-PA diagnosis and photodynamic-photothermal therapeutics within the nanomaterials. Initially, CP5 was modified on the surface of AuNRs to eliminate the cytotoxicity of CTAB. And then, TTPY-Py with AIE effect was encapsulated by CP5-modified AuNRs for fluorescence imaging and efficient ROS generation. Furthermore, the resulting hybrid nanomaterial exhibited reversible and stimuli-responsive fluorescence, benefiting from the 
host-guest interaction. Both in vitro and in vivo experiments demonstrated that TTPY-PyСCP5@AuNR possess excellent biocompatibility and high stability, facilitating the FLI-PA dual imaging-guided and synergistic PDT-PTT. Significantly, TTPY-Py transported by CP5-modified AuNRs could targetedly light up mitochondria after escaping from CP5 and subsequently from lysosomes due to the activation of the acidic environment in lysosomes. This study provides a successful example of supramolecular nanomaterial for multimodal and integrated theranostics, paving a new way to develop hybrid nanotheranostics via facilely supramolecular approaches and opening up promising perspectives to develop new generation of multifunctional phototheranostics.

\section{- ASSOCIATED CONTENT}

\section{Supporting Information}

The Supporting Information is available free of charge on the ACS Publications website.

Synthetic routes and characterizations of compounds, preparation and characterization of materials, ITC experiments to determine the host-guest interactions, controlled experiments of the optical properties, photodynamic and photothermal performance, intracellular total ROS activity assay, CLSM images of 4T1 cells after incubation with pure TTPY-Py, corresponding statistic temperature changes at the tumor sites during the continuous laser irradiation, and supplementary histological and immunohistochemical studies, details for experiments (PDF).

\section{- AUTHOR INFORMATION}

Corresponding Author

wangd@szu.edu.cn 
ywyang@jlu.edu.cn

tangbenz@ust.hk

\section{ORCID}

Nan Song: 0000-0001-6542-1488

Dong Wang: 0000-0001-5137-0771

Ben Zhong Tang: 0000-0002-0293-964X

Notes

The authors declare no competing financial interest.

\section{- ACKNOWLEDGMENT}

This work was funded by the Developmental Fund for Science and Technology of Shenzhen government (Grant No. JCYJ20190808153415062, JCYJ20190808121417291), the China Postdoctoral Science Foundation (2020M672791) the Natural Science Foundation for Distinguished Young Scholars of Guangdong Province (2020B1515020011), and the Natural Science Foundation of China (21801169, 52003164). Natural Science Foundation of Guangdong Province (2018A0303130081) The authors also acknowledge the Instrumental Analysis Center of Shenzhen University. All animal operations complied with the regulations of the Animal Ethical and Welfare Committee of Shenzhen University (AEWC-SZU). The 4T1 mouse breast cancer cell line was purchased from Chinese Academy of Science Cell Bank for Type Culture Collection.

\section{- ABBREVIATIONS}

ACQ: aggregation-caused quenching; AIE: aggregation-induced emission; AIEgens: AIE luminogens; Au NRs: Au nanorods; Baf-A1: Bafilomycin A1; CD31: adhesion molecule-1; CLSM: confocal laser scanning microscopy; CTAB: cetyltrimethylammonium bromide; CP5: carboxylatopillar[5]arene; EPR: enhanced permeability and retention; FDA/PI: fluorescein 
diacetate/propidium iodide; FLI: fluorescene imaging; H\&E: Hematoxylin and eosin; TUNEL: terminal deoxynucleotidyl transferase dUTP nick end labeling; ITC: isothermal calorimetry; LP: long pass; MOFs: metal-organic frameworks; MSNs: mesoporous silica nanoparticles; NIR: near-infrared; PAI: photoacoustic imaging; MRI: magnetic resonance imaging; PDT: photodynamic therapy; PTT: photothermal therapy; Py: pyridinium; ROS: reactive oxygen species; TPA: $p$-toluenesulfonic acid

\section{- REFERENCES}

(1) Song, N.; Zhang, Z.; Liu, P.; Yang, Y.-W.; Wang, L.; Wang, D.; Tang,B. Z.: Nanomaterials with Supramolecular Assembly Based on AIE Luminogens for Theranostic Applications. Adv. Mater. 2020, 2004208.

(2) Huang, J.; He, B.; Zhang, Z.; Li, Y.; Kang, M.; Wang, Y.; Li, K.; Wang, D.; Tang, B. Z.: Aggregation-Induced Emission Luminogens Married to 2D Black Phosphorus Nanosheets for Highly Efficient Multimodal Theranostics. Adv. Mater. 2020, 32, e2003382.

(3) Deng, R.; Shen, N.; Yang, Y.; Yu, H.; Xu, S.; Yang, Y. W.; Liu, S.; Meguellati, K.; Yan, F.: Targeting epigenetic pathway with gold nanoparticles for acute myeloid leukemia therapy. Biomaterials 2018, 167, 80-90.

(4) Zhou, S.; Ding, C.; Wang, Y.; Jiang, W.; Fu, J.: Supramolecular Valves Functionalized Rattle-Structured UCNPs@hm-SiO2 Nanoparticles with Controlled Drug Release Triggered by Quintuple Stimuli and Dual-Modality Imaging Functions: A Potential Theranostic Nanomedicine. ACS Biomater. Sci. \& Engineer. 2019, 5, 6022-6035.

(5) Hu, C.; Ma, N.; Li, F.; Fang, Y.; Liu, Y.; Zhao, L.; Qiao, S.; Li, X.; Jiang, X.; Li, T.; Shen, F.; Huang, Y.; Luo, Q.; Liu, J.: Cucurbit[8]uril-Based Giant Supramolecular Vesicles: Highly Stable, 
Versatile Carriers for Photoresponsive and Targeted Drug Delivery. ACS Appl. Mater. Interfaces 2018, 10, 4603-4613.

(6) Jeong, J.-E.; Uddin, M. A.; Ryu, H. S.; Kim, H.-C.; Kang, M.; Joung, J. F.; Park, S.; Shim, S.-H.; Woo, H. Y.: Green-, Red-, and Near-Infrared-Emitting Polymer Dot Probes for Simultaneous Multicolor Cell Imaging with a Single Excitation Wavelength. Chem. Mater. 2020, $32,6685-6696$.

(7) Li, Y.; Wu, Q.; Kang, M.; Song, N.; Wang, D.; Tang, B. Z.: Boosting the photodynamic therapy efficiency by using stimuli-responsive and AIE-featured nanoparticles. Biomaterials 2020, $232,119749$.

(8) Chen, Y.; Lu, W.; Guo, Y.; Zhu, Y.; Song, Y.: Chitosan-Gated Fluorescent Mesoporous Silica Nanocarriers for the Real-Time Monitoring of Drug Release. Langmuir 2020, 36, 6749-6756.

(9) Yang, J.; Yang, Y. W.: Metal-Organic Frameworks for Biomedical Applications. Small 2020, $16, \mathrm{e} 1906846$.

(10) Li, M.; Bai, H.; Shao, L.; Hua, B.: A Multifunctional Hybrid[4]arene-Based Macrocyclic Amphiphile: Self-Assembly, Tunable LCST Behavior, and Construction of Fluorescent Nanoparticles for Cell Imaging. Org. Lett. 2019, 21, 8943-8947.

(11) Li, Z.; Song, N.; Yang, Y.-W.: Stimuli-Responsive Drug-Delivery Systems Based on Supramolecular Nanovalves. Matter 2019, 1, 345-368.

(12) Lou, X. Y.; Li, Y. P.; Yang, Y. W.: Gated Materials: Installing Macrocyclic Arenes-Based Supramolecular Nanovalves on Porous Nanomaterials for Controlled Cargo Release. Biotechnol. J 2019, 14, e1800354.

(13) Song, N.; Yang, Y.-W.: Molecular and supramolecular switches on mesoporous silica 
nanoparticles. Chem. Soc. Rev. 2015, 44, 3474-3504.

(14) Ha Lien, N. T.; Phan, A. D.; Van Khanh, B. T.; Thuy, N. T.; Trong Nghia, N.; My Nhung, H. T.; Hong Nhung, T.; Quang Hoa, D.; Duong, V.; Minh Hue, N.: Applications of Mesoporous Silica-Encapsulated Gold Nanorods Loaded Doxorubicin in Chemo-photothermal Therapy. ACS Omega 2020, 5, 20231-20237.

(15) Ma, B.; Xu, H.; Zhuang, W.; Wang, Y.; Li, G.: Reactive Oxygen Species Responsive Theranostic Nanoplatform for Two-Photon Aggregation-Induced Emission Imaging and Therapy of Acute and Chronic Inflammation. ACS Nano 2020, 14, 5862-5873.

(16) Yang, S.; You, Q.; Yang, L.; Li, P.; Lu, Q.; Wang, S.; Tan, F.; Ji, Y.; Li, N.: Rodlike MSN@Au Nanohybrid-Modified Supermolecular Photosensitizer for NIRF/MSOT/CT/MR Quadmodal Imaging-Guided Photothermal/Photodynamic Cancer Therapy. ACS Appl. Mater. Interfaces 2019, 11, 6777-6788.

(17) Ma, Z.; Zhang, Y.; Zhang, J.; Zhang, W.; Foda, M. F.; Dai, X.; Han, H.: Ultrasmall Peptide-Coated Platinum Nanoparticles for Precise NIR-II Photothermal Therapy by Mitochondrial Targeting. ACS Appl. Mater. Interfaces 2020, 12, 39434-39443.

(18) Li, H.; Wei, R.; Yan, G. H.; Sun, J.; Li, C.; Wang, H.; Shi, L.; Capobianco, J. A.; Sun, L.: Smart Self-Assembled Nanosystem Based on Water-Soluble Pillararene and Rare-Earth-Doped Upconversion Nanoparticles for pH-Responsive Drug Delivery. ACS Appl. Mater. Interfaces 2018, $10,4910-4920$.

(19) Kim, D. H.; Kim, D. W.; Jang, J. Y.; Lee, N.; Ko, Y. J.; Lee, S. M.; Kim, H. J.; Na, K.; Son, S. U.: Fe3O4@Void@Microporous Organic Polymer-Based Multifunctional Drug Delivery Systems: Targeting, Imaging, and Magneto-Thermal Behaviors. ACS Appl. Mater. Interfaces 2020, 
$12,37628-37636$.

(20) Wang, L.; Yang, P.-P.; Zhao, X.-X.; Wang, H.: Self-assembled nanomaterials for photoacoustic imaging. Nanoscale 2016, 8, 2488-2509.

(21) Cheng, H.-B.; Li, Y.; Tang, B. Z.; Yoon, J.: Assembly strategies of organic-based imaging agents for fluorescence and photoacoustic bioimaging applications. Chem. Soc. Rev. 2020, $49,21-31$

(22) Zhao, L.; Liu, Y.; Xing, R.; Yan, X.: Supramolecular Photothermal Effects: A Promising Mechanism for Efficient Thermal Conversion. Angew. Chem. Int. Ed. 2020, 59, 3793-3801.

(23) Zhang, Z.; Xu, W.; Kang, M.; Wen, H.; Guo, H.; Zhang, P.; Xi, L.; Li, K.; Wang, L.; Wang, D.; Tang, B. Z.: An All-Round Athlete on the Track of Phototheranostics: Subtly Regulating the Balance between Radiative and Nonradiative Decays for Multimodal Imaging-Guided Synergistic Therapy. Adv. Mater. 2020, e2003210.

(24) Qin, W.; Ding, D.; Liu, J.; Yuan, W. Z.; Hu, Y.; Liu, B.; Tang, B. Z.: Biocompatible Nanoparticles with Aggregation-Induced Emission Characteristics as Far-Red/Near-Infrared Fluorescent Bioprobes for In Vitro and In Vivo Imaging Applications. Adv. Funct. Mater. 2012, 22, 771-779.

(25) Wang, Q.; Zhang, P.; Xu, J.; Xia, B.; Tian, L.; Chen, J.; Li, J.; Lu, F.; Shen, Q.; Lu, X.; Huang, W.; Fan, Q.: NIR-Absorbing Dye Functionalized Supramolecular Vesicles for Chemo-photothermal Synergistic Therapy. ACS Appl. Bio. Mater. 2018, 1, 70-78.

(26) Chen, Y. S.; Zhao, Y.; Yoon, S. J.; Gambhir, S. S.; Emelianov, S.: Miniature gold nanorods for photoacoustic molecular imaging in the second near-infrared optical window. Nat. Nanotechnol. 2019, 14, 465-472. 
noncovalently tailorable surface for multi- modality image-guided chemo-photothermal cancer therapy. Chem. Commun. 2019, 55, 13506-13509.

(28) Hauwiller, M. R.; Ye, X.; Jones, M. R.; Chan, C. M.; Calvin, J. J.; Crook, M. F.; Zheng, H.; Alivisatos, A. P.: Tracking the Effects of Ligands on Oxidative Etching of Gold Nanorods in Graphene Liquid Cell Electron Microscopy. ACS Nano 2020, 14, 10239-10250.

(29) Zhou, J.; Tian, F.; Fu, R.; Yang, Y.; Jiao, B.; He, Y.: Enzyme-Nanozyme Cascade Reaction-Mediated Etching of Gold Nanorods for the Detection of Escherichia coli. ACS Appl. Nano Mater. 2020, 3, 9016-9025.

(30) Wang, D.; Tang, B. Z.: Aggregation-Induced Emission Luminogens for Activity-Based Sensing. Acc. Chem. Res. 2019, 52, 2559-2570.

(31) Mei, J.; Leung, N. L.; Kwok, R. T.; Lam, J. W.; Tang, B. Z.: Aggregation-Induced Emission: Together We Shine, United We Soar! Chem. Rev. 2015, 115, 11718-940.

(32) Xia, F.; Wu, J.; Wu, X.; Hu, Q.; Dai, J.; Lou, X.: Modular Design of Peptide- or DNA-Modified AIEgen Probes for Biosensing Applications. Acc. Chem. Res. 2019, 52, 3064-3074.

(33) Zhang, R.; Niu, G.; Lu, Q.; Huang, X.; Chau, J. H. C.; Kwok, R. T. K.; Yu, X.; Li, M.-H.; Lam, J. W. Y.; Tang, B. Z.: Cancer cell discrimination and dynamic viability monitoring through wash-free bioimaging using AIEgens. Chem. Sci. 2020, 11,7676-7684.

(34) Han, T.; Yan, D.; Wu, Q.; Song, N.; Zhang, H.; Wang D.: Aggregation-Induced Emission: A Rising Star in Chemistry and Materials Science. Chin. J. Chem. 2020, 38, doi: 10.1002/cjoc.202000520. 
AIE photosensitizer for simultaneous bioimaging and photodynamic therapy. Mater. Chem. Front. 2020, 4, 3074-3085.

(36) Zhao, Z.; Zhang, H.; Lam, J. W. Y.; Tang, B. Z.: Aggregation-Induced Emission: New Vistas at Aggregate Level. Angew. Chem. Int. Ed. 2020, 59, 9888-9907.

(37) Gao, M.; Tang, B. Z.: AIE-based cancer theranostics. Coord. Chem. Rev. 2020, 402, 213076.

(38) Liu, S.; Ou, H.; Li, Y.; Zhang, H.; Liu, J.; Lu, X.; Kwok, R. T. K.; Lam, J. W. Y.; Ding, D.; Tang, B. Z.: Planar and Twisted Molecular Structure Leads to the High Brightness of Semiconducting Polymer Nanoparticles for NIR-II Fluorescence Imaging. J. Am. Chem. Soc. 2020, $142,15146-15156$.

(39) Xu, W.; Zhang, Z.; Kang, M.; Guo, H.; Li, Y.; Wen, H.; Lee, M. M. S.; Wang, Z.; Kwok, R. T. K.; Lam, J. W. Y.; Li, K.; Xi, L.; Chen, S.; Wang, D.; Tang, B. Z.: Making the Best Use of Excited-State Energy: Multimodality Theranostic Systems Based on Second Near-Infrared (NIR-II) Aggregation-Induced Emission Luminogens (AIEgens). ACS Mater. Lett. 2020, 2, 1033-1040.

(40) Chen, H.; Zeng, X.; Tham, H. P.; Phua, S. Z. F.; Cheng, W.; Zeng, W.; Shi, H.; Mei, L.; Zhao, Y.: NIR-Light-Activated Combination Therapy with a Precise Ratio of Photosensitizer and Prodrug Using a Host-Guest Strategy. Angew. Chem. Int. Ed. 2019, 58, 7641-7646.

(41) Cui, W.; Wang, L.; Xu, L.; Zhang, G.; Meier, H.; Tang, H.; Cao, D.: Fluorescent-Cavity Host: An Efficient Probe to Study Supramolecular Recognition Mechanisms. J. Phys. Chem. Lett. 2018, 9, 1047-1052.

(42) Feng, H. T.; Li, Y.; Duan, X.; Wang, X.; Qi, C.; Lam, J. W. Y.; Ding, D.; Tang, B. Z.: 
Substitution Activated Precise Phototheranostics through Supramolecular Assembly of AIEgen and Calixarene. J. Am. Chem. Soc. 2020, 142, 15966-15974.

(43) Lei, S. N.; Xiao, H.; Zeng, Y.; Tung, C. H.; Wu, L. Z.; Cong, H.: BowtieArene: A Dual Macrocycle Exhibiting Stimuli-Responsive Fluorescence. Angew. Chem. Int. Ed. 2020, 59, 10059-10065.

(44) Shi, X.; Zhang, X.; Ni, X.-L.; Zhang, H.; Wei, P.; Liu, J.; Xing, H.; Peng, H.-Q.; Lam, J. W. Y.; Zhang, P.; Wang, Z.; Hao, H.; Tang, B. Z.: Supramolecular Polymerization with Dynamic Self-Sorting Sequence Control. Macromolecules 2019, 52, 8814-8825.

(45) Yang, X.; Mao, W.; Liu, Y.; Li, L.; Ma, D.: Supramolecular Vesicles Based on Water-Soluble 2,6-Helic[6]arene: High Affinity Binding, Stimuli Responsiveness and Delivery of Doxorubicin to Cancer Cells. Chin. J. Chem. 2019, 37, 575-580.

(46) Chen, C.; Ni, X.; Tian, H.-W.; Liu, Q.; Guo, D.-S.; Ding, D. Nonradiative Decay and Intersystem Crossing for Ultrasensitive Fluorescence Image-Guided Cancer Surgery. Angew. Chem. Int. Ed. 2020, 59, 10008-10012.

(47) Li, J.; Wang, J.; Li, H.; Song, N.; Wang, D.; Tang, B. Z.: Supramolecular materials based on AIE luminogens (AIEgens): construction and applications. Chem. Soc. Rev. 2020, 49, $1144-1172$.

(48) Lan, S.; Liu, Y.; Shi, K.; Ma, D.: Acetal-Functionalized Pillar[5]arene: A pH-Responsive and Versatile Nanomaterial for the Delivery of Chemotherapeutic Agents. ACS Appl. Bio. Mater. 2020, 3, 2325-2333.

(49) Li, C.; Xie, Z.; Chen, Q.; Zhang, Y.; Chu, Y.; Guo, Q.; Zhou, W.; Liu, P.; Chen, H.; Jiang, C.; Sun, K.; Sun, T.: Supramolecular Hunter Stationed on Red Blood Cells for Detoxification 
Based on Specific Molecular Recognition. ACS Nano 2020, 14, 4950-4962.

(50) Song, N.; Lou, X. Y.; Ma, L.; Gao, H.; Yang, Y. W.: Supramolecular nanotheranostics based on pillarenes. Theranostics 2019, 9, 3075-3093.

(51) Yu, G.; Wu, D.; Li, Y.; Zhang, Z.; Shao, L.; Zhou, J.; Hu, Q.; Tang, G,; Huang, F.: A pillar[5]arene-based [2]rotaxane lights up mitochondria. Chem. Sci. 2016, 7, 3017-3024.

(52) Song, N.; Kakuta, T.; Yamagishi, T.-a.; Yang, Y.-W.; Ogoshi, T.: Molecular-Scale Porous Materials Based on Pillar[n]arenes. Chem 2018, 4, 2029-2053.

(53) Wang, X. H.; Song, N.; Hou, W.; Wang, C. Y.; Wang, Y.; Tang, J.; Yang, Y. W.: Efficient Aggregation-Induced Emission Manipulated by Polymer Host Materials. Adv. Mater. 2019, 31, e1903962.

(54) Xin, P.; Kong, H.; Sun, Y.; Zhao, L.; Fang, H.; Zhu, H.; Jiang, T.; Guo, J.; Zhang, Q.;

Dong, W.; Chen, C. P.: Artificial K(+) Channels Formed by Pillararene-Cyclodextrin Hybrid Molecules: Tuning Cation Selectivity and Generating Membrane Potential. Angew. Chem. Int. Ed. 2019, 58, 2779-2784.

(55) Ogoshi, T.; Kanai, S.; Fujinami, S.; Yamagishi, T.; Nakamoto, Y.: para-Bridged Symmetrical Pillar[5]arenes: Their Lewis Acid Catalyzed Synthesis and Host-Guest Property. $J$. Am. Chem. Soc. 2008, 130, 5022-5023.

(56) Muhammed, M. A. H.; Cruz, L. K.; Emwas, A. H.; El-Zohry, A. M.; Moosa, B.; Mohammed, O. F.; Khashab, N. M.: Pillar[5]arene-Stabilized Silver Nanoclusters: Extraordinary Stability and Luminescence Enhancement Induced by Host-Guest Interactions. Angew. Chem. Int. Ed. 2019, 58, 15665-15670.

(57) Shi, B.; Jie, K.; Zhou, Y.; Zhou, J.; Xia, D.; Huang, F.: Nanoparticles with Near-Infrared 
Emission Enhanced by Pillararene-Based Molecular Recognition in Water. J. Am. Chem. Soc.

2016, 138, 80-3.

(58) Yang, J. L.; Yang, Y. H.; Xun, Y. P.; Wei, K. K.; Gu, J.; Chen, M.; Yang, L. J.: Novel Amino-pillar[5]arene as a Fluorescent Probe for Highly Selective Detection of Au(3+) Ions. ACS Omega 2019, 4, 17903-17909.

(59) Chang, Y.; Chen, J. Y.; Yang, J.; Lin, T.; Zeng, L.; Xu, J. F.; Hou, J. L.; Zhang, X.: Targeting the Cell Membrane by Charge-Reversal Amphiphilic Pillar[5]arene for the Selective Killing of Cancer Cells. ACS Appl. Mater. Interfaces 2019, 11, 38497-38502.

(60) Liu, Y.; Liao, Y.; Li, P.; Li, Z. T.; Ma, D.: Cross-Linked Pillar[6]arene Nanosponges Fabricated by the Use of a Supra-Amphiphilic Template: Cargo Encapsulation and Overcoming Multidrug Resistance. ACS Appl. Mater. Interfaces 2020, 12, 7974-7983.

(61) Shang, K.; Wang, Y.; Lu, Y.; Pei, Z.; Pei, Y.: Dual-Targeted Supramolecular Vesicles Based on the Complex of Galactose Capped Pillar[5]Arene and Triphenylphosphonium Derivative for Drug Delivery. Isr. J. Chem. 2018, 58, 1241-1245.

(62) Yu, G.; Tang, G.; Huang, F.: Supramolecular enhancement of aggregation-induced emission and its application in cancer cell imaging. J. Mater. Chem. C 2014, 2, 6609-6617.

(63) Shao, L.; Pan, Y.; Hua, B.; Xu, S.; Yu, G.; Wang, M.; Liu, B.; Huang, F.: Constructing Adaptive Photosensitizers via Supramolecular Modification Based on Pillararene Host-Guest Interactions. Angew. Chem. Int. Ed. 2020, 59, 11779-11783.

(64) Roopa, Kumar, N.; Bhalla, V.; Kumar, M.: Development and sensing applications of fluorescent motifs within the mitochondrial environment. Chem. Commun. 2015, 51, $15614-15628$. 
Plasmon Resonant Gold Nanoparticles. J. Phys. Chem. C 2007, 111, 3636-3641.

(66) Tan, J.; Namuangruk, S.; Kong, W.; Kungwan, N.; Guo, J.; Wang, C.: Manipulation of Amorphous-to-Crystalline Transformation: Towards the Construction of Covalent Organic Framework Hybrid Microspheres with NIR Photothermal Conversion Ability. Angew. Chem. Int. Ed. 2016, 55, 13979-13984.

(67) Nikoobakht, B.; El-Sayed, M. A.: Preparation and Growth Mechanism of Gold Nanorods (NRs) Using Seed-Mediated Growth Method. Chem. Mater. 2003, 15, 1957-1962.

(68) Wang, D.; Lee, M. M. S.; Shan, G.; Kwok, R. T. K.; Jacky W. Y. Lam, Su, H.; Cai, Y.; Tang, B. Z.: Highly Efficient Photosensitizers with Far-Red/Near-Infrared Aggregation-Induced Emission for In Vitro and In Vivo Cancer Theranostics. Adv. Mater. 2018, 30, 1802105.

\section{Table of Contents Graphic}

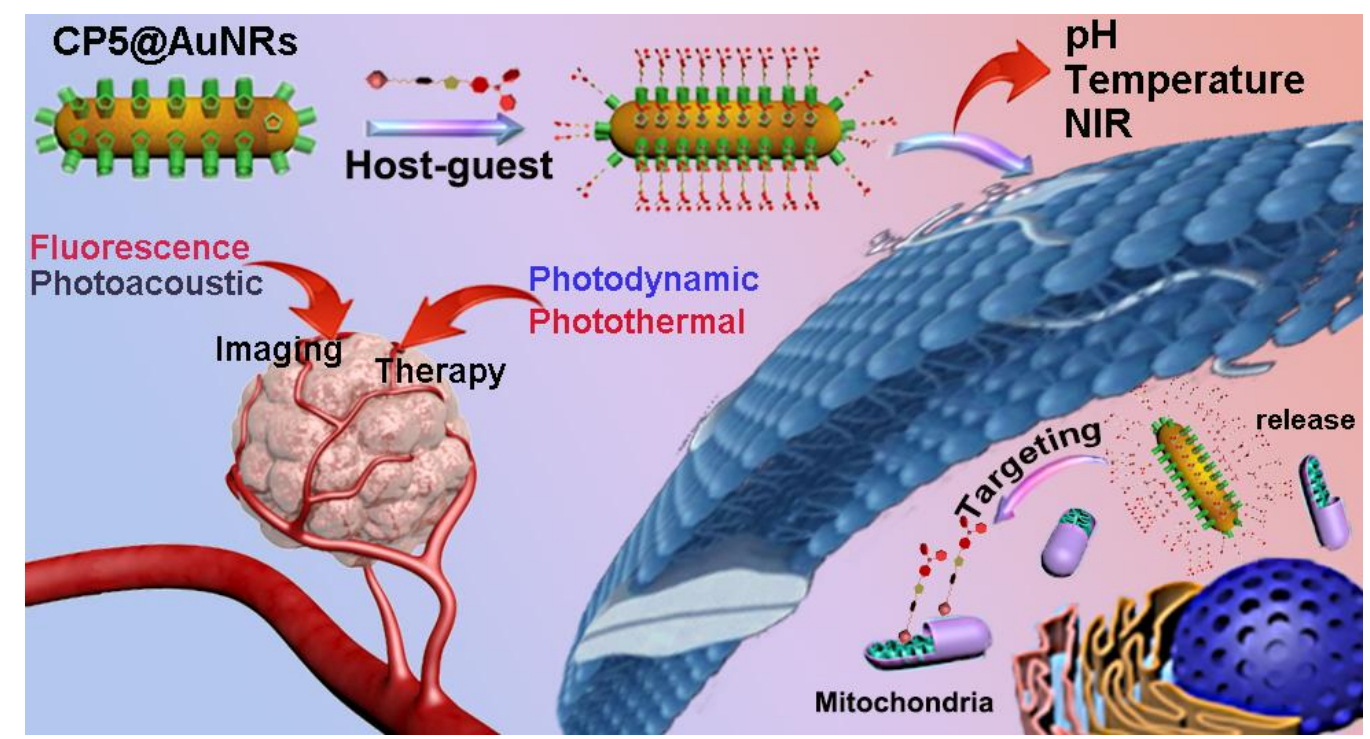

